\title{
MIOCENE TO RECENT RADIOLARIANS FROM SOUTHERN PACIFIC COAST OF COSTA RICA
}

\author{
RADIOLARIOS DEL MIOCENO AL RECIENTE DEL PACÍFICO SUR DE COSTA RICA
}

\author{
María I. Sandoval \\ Escuela Centroamericana de Geología, Universidad de Costa Rica \\ mariaisabel.sandoval@ucr.ac.cr
}

(Recibido: 04/06/2017; aceptado: 06/09/2017)

\begin{abstract}
Radiolarians from Miocene-Recent marine sediments are taxonomically reported for the first time in Costa Rica using samples from Sites U1381C and U1414 A of the Integrated Ocean Drilling Program CRISP A2 expedition. Both sites are presently located offshore Osa Peninsula, northeastern equatorial Pacific. The sediments retrieved from the two sites mainly consist of nannofossil calcareous ooze with foraminifera, sponge spicules, diatoms and radiolarians. In both sequences, radiolarians vary from absent to abundant and well preserved. This work presents the systematic paleontology of the radiolarians recorded including taxonomic notes and illustrations of the species. An associated publication provides the statistic and biostratigraphic data for the sites, spanning from the middle Miocene to the Recent (radiolarian zones RN5 to RN16). In total, 116 species (14 families and 41 genera) were found. Hole U1414 A yielded a total of 105 species and 65 taxa with conditional identifications ("species groups"), and Hole U1381C yielded a total of 90 species and 55 species groups.

Keywords: Radiolaria Polycystina, Cocos Plate, Cenozoic, Miocene, IODP 344 Expedition, Costa Rica, Eastern Equatorial Pacific.

RESUMEN: El presente estudio reporta por primera vez la taxonomía de radiolarios pertenecientes a sedimentos marinos de edad Mioceno-Reciente de Costa Rica. La identificación taxonómica se realizó utilizando los sedimentos obtenidos en las perforaciones U1381C y U1414 A de la Expedición CRISP A2 del Programa Integral de Perforación Oceánica, (IODP, por sus siglas en inglés). Ambos sitios están ubicados en mar abierto cercanos a la Península de Osa, Pacífico este ecuatorial. Dichos sedimentos consisten en un ooze calcáreo con nanofósiles, foraminíferos, diatomeas, espículas de esponja y radiolarios. En ambas secuencias, la recuperación de radiolarios varía desde ausentes a abundantes y bien preservados. El siguiente estudio presenta la sistemática paleontológica a detalle junto con las ilustraciones
\end{abstract}


de radiolarios. Una publicación previa asociada a este estudio proporciona los datos estadísticos y bioestratigráficos de los sitios, los cuales corresponden al Mioceno medio-Reciente (zonación RN5 a RN16). El número total de especies identificadas en las muestras es de 116, distribuidas en 14 familias y 41 géneros. La perforación U1414 A suma un total de 105 especies y 65 taxones con identificaciones condicionales (grupos), y la perforación U1381C un total de 90 especies y 55 grupos.

Palabras clave: Radiolaria Polycystina, placa Cocos, Cenozoico, Mioceno, Expedición 344, Costa Rica, Pacífico Este Ecuatorial.

\section{INTRODUCTION}

Polycystine Radiolaria is a major group of marine planktonic protists. Their skeletons are composed of amorphous silica, allowing their preservation in the sediments. In Costa Rica, radiolarians are the oldest fossils present in the geological record. Neogene radiolarians from the sedimentary cover of the Cocos plate are the subject of the present study. Their taxonomy and biostratigraphy is based on continuously cored deep-sea successions. Radiolarian data have been correlated with that of other microfossils, such as calcareous nannoplankton and foraminifers, and compared with geomagnetic polarity, in order to constrain the ages of the deposits.

During the last forty years, several authors have contributed to the development of regional stratigraphic radiolarian zonations for the Equatorial Pacific area (Riedel, 1957, 1959; Nigrini, 1971; Moore, 1971; Riedel and Sanfilippo 1971, 1978; Goll, 1980). Radiolarians have also been used for paleoenvironmental reconstructions, identifying tropical, subtropical, cool temperate, polar and upwelling assemblages (Moore, van Andel, Sancetta \& Pisias, 1978). The association of radiolarian assemblages with water masses (differing chiefly in temperature and nutrients) has been described from many areas, including, among others, the Pacific Ocean (Nigrini, 1970; Riedel, 1971a; Sachs, 1973; Renz, 1976; MolinaCruz, 1977; Boltovskoy and Jankilevich, 1985; Boltovskoy and Riedel, 1987), the Indian Ocean (Johnson and Nigrini, 1980, 1982) and the Atlantic Ocean (Bjørklund, Cotesea, Swanberg \& Schrader, 1998; Boltovskoy, 1998, 1999).
Thus, radiolarian fossil remains can also provide valuable clues to the ecological settings (primarily temperature and productivity) of the overlying waters at the time when these populations dwelled in the water-column. Although this technique, as most others, has its shortcomings (Boltovskoy, 1994; Haslett, 2002), it has proven its usefulness in a numbers of detailed surveys world-wide.

Since the end of the 19th century, several high-level taxonomic schemes have been proposed for the polycystines. Haeckel (1887) produced one of the earliest comprehensive systems of radiolarian classification, describing thousands of new polycystine species. Haeckel's work is still a necessary reference guide, but it does not satisfactorily represent natural relationships, because groupings are only based on morphologic similarities of the skeleton only, and because the rigidity of these geometry-based diagnoses often ignores the ample intraspecific variability of these protists. Efforts to improve the classification schemes inherited from earlier workers have followed two different main approaches, which are cytological data and evolutionary studies. Several revisions that rely heavily on cytoplasmic features have been proposed (Hollande and Enjumet, 1960; Cachon and Cachon, 1972a, b; Petrushevskaya, Cachon \& Cachon, 1976; Petrushevskaya, 1981). Although these schemes are probably sounder in biological terms, their application to fossil and subfossil materials lacking the protoplasm is problematic, which is one of the reasons for their very limited acceptance among radiolarian workers. Analyses of evolutionary lineages in geological sequences were used as a basis to assess the taxonomic value of key skeletal traits; it was concluded that 
many of them (e.g. number of segments, number of supplementary concentric spheres, number of feet, number of rays and of equatorial spines in discoidal Spumellaria, presence and nature of thoracic wings) have little or no suprageneric value. In contrast, several others (e.g. cephalic structure, pore arrangement, shell terminations in Nassellaria), traditionally considered as of minor value, are conservative through time and reveal evolutionary lineages and, therefore, are relevant for higher-rank divisions (Riedel and Sanfilippo, 1986). Based on skeletal features only, several authors proposed alternative classifications, either for the entire order or for selected polycystine groups (Riedel, 1967a in Banner et al., 1967, 1971b; Petrushevskaya, 1965, 1971; Goll, 1968, 1969; Sanfilippo and Riedel, 1970; Dumitrica, 1989; De Wever, Dumitrica, Caulet, Nigrini \& Caridroit, 2001). The presently most widely accepted classification for extant and Cenozoic radiolarians is still that of Riedel (1967a) and Riedel (1971b), which is adopted in this work. The clasification of Miocene polycystines is based on De Wever et al. (2001).

This study is based on open ocean drill sites and intends to provide the first detailed MioceneRecent radiolarian inventory of Pacific sediments off Costa Rica. As opposed to most previous works in the area, especially those oriented at paleoceanographic reconstructions (see references above), which used restricted subsets of the fauna (usually 20-40 morphotypes), it covers all the radiolarians found in the samples.

\section{GEOLOGICAL SETTING}

The western active margin off Costa Rica represents the subduction zone resulting from the interaction of the Cocos plate and the Caribbean plate. The drilled sites of the expedition CRISP A2 are located near the interaction between the two plates, with the objective to characterize the rocks and sediments of the plate's surrounds (Fig. 1). Hole U1381C is located about $4.5 \mathrm{~km}$ seaward of the accretionary prism, offshore the Osa Peninsula, on the incoming Cocos plate $\left(8^{\circ} 25.7027^{\prime} \mathrm{N}, 84^{\circ} 9.4805^{\prime} \mathrm{W}, 2064.6 \mathrm{~m}\right.$ water depth). Hole U1414 A is located on the incoming Cocos plate, about $1 \mathrm{~km}$ seaward of the deformation front $\left(8^{\circ} 30.2304^{\prime} \mathrm{N}, 84^{\circ} 13.5298^{\prime} \mathrm{W}, 2459 \mathrm{~m}\right.$ water depth) (Fig. 1). The hole U1381C is $109 \mathrm{~m}$ long and is dominated by pelagic and hemipelagic sediments intercalated by numerous tephra layers and a basaltic breccia layers at the bottom. The detail of the units is described by Harris et al. (2013) and Schindlbeck et al. (2015, 2016). The Hole U1414 A measures $471.6 \mathrm{~m}$, including $375.25 \mathrm{~m}$ of sedimentary deposits and $96.35 \mathrm{~m}$ of oceanic basaltic basement. The biogenic fraction mainly consists of calcareous nannofossil-rich ooze with foraminifers, diatoms, sponge spicules and radiolarians.

\section{MATERIAL AND METHODS}

Two cores of the Integrated Ocean Drilling Program (IODP) Expedition 344: Costa Rica Seismogenesis Project, were sampled. The two cores contain calcareous nannofossil-rich ooze with foraminifera, diatoms and radiolarians. Two hundred and ninety-five samples were prepared (69 from Hole U1381C and 226 from Hole U1414 A) using the following standard procedure methods for Neogene sediments (De Wever, Dumitrica, Caulet, Nigrini \& Caridroit, 2001): (1) Sediments (10-20 g) were placed in beakers with $100-150 \mathrm{~mL}$ of $10 \%$ hydrogen peroxide. (2) Samples were boiled for a few minutes to achieve oxidation of the organic matter and disaggregation of the clay fraction. (3) Samples were rinsed with water and sieved through a $60 \mu \mathrm{m}$ mesh. (4) The wet samples were placed again in beakers adding of a few drops of $10 \%$ hydrochloric acid to eliminate foraminifers, coccolithophorids, and other calcareous remains. (5) The samples were rinsed and sieved again through a $60 \mu \mathrm{m}$ mesh. (6) A drop of the wet residue $(1 \mathrm{~mL})$ was placed onto a labeled glass slide, dried for a few minutes on a hot plate, embedded in Norland 60 mounting medium, and covered with a coverslip. (7) The slides were exposed for some minutes to ultraviolet light until dry. (8) The mounted samples were analyzed under a transmitted light microscope. 


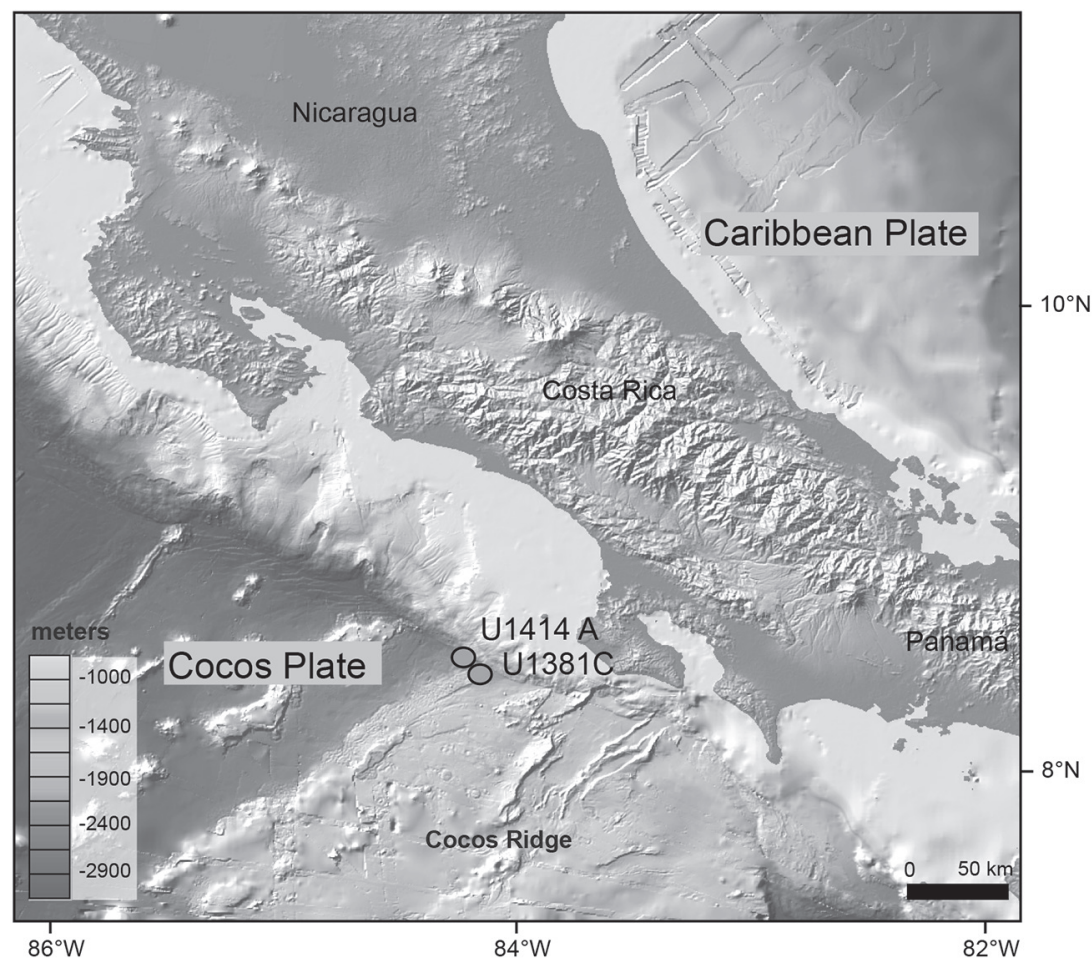

Figure 1: Location of of Holes U1414 A and U1381C from IODP Expedition 344 in the geological context of Costa Rica. The topographic map is adapted from www.geomapapp.org (Ryan et al., 2009).

The age model was established using radiolarian data (Sandoval, Boltovskoy, Baxter \& Baumgartner, 2017), preliminary nannofossil shipboard data (Harris et al. 2013) and published tephra dating with 40Ar/39Ar (Schindlbeck et al., 2015, 2016).

Images of radiolarians were taken with an Olympus XC10 camera. In many cases, several photographs of the same specimen were obtained and processed with the aid of the Image J program (Extended Depth of Field), which allows merging a stack of photos in different focal positions into a single composite image (Forster, van de Ville, Berent, Sage \& Unser, 2004).

\section{SYSTEMATIC PALEONTOLOGY}

Class ACTINOPODA Calkins, 1909

Subclass RADIOLARIA Müller, 1858

Superorder POLYCYSTINA Ehrenberg, 1838, emend. Riedel, 1967a in Banner et al., 1967
Order SPUMELLARIA Ehrenberg, 1875,

emend. Riedel, 1971a

Family COLLOSPHAERIDAE Müller, 1858

\section{Genus Acrosphaera Haeckel, 1881}

Acrosphaera sp. A (Fig. 2A)

Remarks: Regular sphere, covered with numerous, straight, radial spines, irregularly scattere over the whole surface. It presents some protuberances and depressions. Dimensions: $\sim 100 \mu \mathrm{m}$ in diameter, spines $20-40 \mu \mathrm{m}$.

\section{Acrosphaera sp. B (Fig. 2B)}

Remarks: Sub-circular sphere covered with numerous irregular pores. The size and shape of the spines is irregular. Dimensions: $\sim 150-170 \mu \mathrm{m}$ in diameter, spines $20-50 \mu \mathrm{m}$, pores size $10-50 \mu \mathrm{m}$.

Acrosphaera murrayana (Haeckel) (Fig. 2C) Choenicosphaera murrayana n. sp. Haeckel, 1887, p. 102, pl. 8, fig. 4. 
Polysolenia murrayana (Haeckel) - Nigrini, 1968, p. 52, pl. 1, figs. 1a-b - Nigrini and Moore, 1979, pl. 2, figs. 4a-b.

Acrosphaera murrayana (Haeckel) -

Takahashi, 1991, pl. 1, figs. 6-10.

\section{Genus Siphonosphaera Müller, 1858}

Siphonosphaera martensi Brandt (Figs. 2D and 2E)

Siphonosphaera martensi n.sp. Brandt, 1905, p. 339, pl. 9, figs. 9-12 - Boltovskoy and Riedel, 1980, p. 104, pl. 1, fig. 8 - Takahashi, 1991, p. 59, pl. 4, figs. 4-5, 7-8.

Remarks: Circular sphere covered with numerous irregular polygonal pores. Surface is gently sharped. Dimensions: $\sim 100-130 \mu \mathrm{m}$ in diameter, pores size $10-25 \mu \mathrm{m}$.

Siphonosphaera polysiphonia Haeckel (Figs. $2 \mathrm{~F}$ and $2 \mathrm{G})$

Siphonosphaera polysiphonia n.sp. Haeckel, 1887, p. 106 - Nigrini 1967, p. 18-19, pl. 1, figs. 4a-b.

\section{Genus Collosphera Müller, 1855}

Collosphaera macropora Popofsky (Figs. 2H, 2I, 2J and 2K)

Collosphaera macropora n.sp. Popofsky, 1914, p. 247, pl. 14, fig. 2 a-c - Boltovskoy and Riedel, 1980, p. 103, pl. 1, fig. 6 - Takahashi, 1991, p. 56, pl. 2, figs. 13-18.

\section{Collosphaera sp.A (Fig. 2L)}

Remarks: Spherical to sub-spherical sphere surrounded by numerous sub-circular pores, irregular arrangement of pores. In general the pores are small, it varies from $\sim 20$ to $5 \mu \mathrm{m}$. Few pointed spines. Dimension: $\sim 150 \mu \mathrm{m}$ in diameter.

Collosphaera elliptica Chen and Tan (Fig. 2M)

Collosphaera elliptica n.sp. Chen and Tan, 1989, p. 1, pl. 1, figs. 1-2 - Tan and Chen, 1999, p. 132, figs. 5-27.

Collosphaera tuberosa Haeckel (Fig. 2N)

Collosphaera tuberosa n.sp. Haeckel, 1887, p. 97 - Molina-Cruz, 1977, pl. 2, fig. 6 Boltovskoy and Riedel, 1980, p. 104, pl. 1, fig. 7 - Takahashi and Honjo, 1981, p. 144, pl. 1, fig. 2.

\section{Genus Otosphaera Haeckel, 1887}

Otosphaera polymorpha Haeckel (Figs. 2O, 2P and 2Q)

Otosphaera polymorpha Haeckel, 1887, p. 116, pl. 7, fig. 6 - Nigrini and Moore, 1979, p. S9, pl. 1 , fig. 5 .

Otosphaera aff. auriculata Haeckel, 1887 (Figs. 2R and 2S)

aff. Haeckel, 1887, p.116, pl.7, fig.5

Remarks: The shell bears three short tubes with perforated walls whose distal end is circular. Pores are very small, irregular shape and arrangement. Dimensions: $\sim 100 \mu \mathrm{m}$, pores $1-5 \mu \mathrm{m}$.

\section{Genus Trisolenia Haeckel, 1881}

Trisolenia zanguebarica Ehrenberg (Fig. 2T)

Trisolenia zanguebarica n.sp. Ehrenberg, 1872 a, p. 321.

Family ACTINOMMIDAE Haeckel, 1862, emend. Riedel, 1967a in Banner et al., 1967

\section{Genus Actinomma Haeckel, 1862}

Actinomma langii (Dreyer) (Figs. 3A and 3B)

Sphaeropyle langii Dreyer, 1889, p. 89, fig. 54 - Foreman, 1975 , p. 618, pl. 9, figs. 30-31 Suzuki, 2006, p. 861, fig. 13.

\section{Genus Cladococcus Haeckel, 1860}

Cladococcus cervicornis Haeckel (Fig. 3C)

Cladococcus cervicornis Haeckel, 1860, p. 801 - Boltovskoy and Riedel, 1980, p. 108, pl. 2, fig. 5 .

\section{Genus Diartus Sanfilippo and Riedel, 1980} 3D)

Diartus hughesi (Campbell and Clark) (Fig.

Ommatocampe (Ommatocampula) hughesi n.sp. Campbell and Clark, 1944, p. 23, pl. 3, fig. 12.

Diartus hughesi (Campbell and Clark) Sanfilippo and Riedel, 1980, p. 1010 - Nigrini and Sanfilippo, 2001, p. 221.

Diartus petterssoni Riedel and Sanfilippo (Figs. 3E and 3F) 
Cannartus (?) petterssoni Riedel and Sanfilippo, 1970, p. 520, pl. 14, fig. 3.

Diartus petterssoni (Riedel and Sanfilippo) Sanfilippo and Riedel, 1980, p. 1010 - Nigrini and Sanfilippo, 2001, p. 223.

\section{Genus Didymocyrtis Haeckel, 1862}

Didymocyrtis penultima (Riedel) (Fig. 3G)

Panarium penultimum Riedel, 1957, p. 76, pl. 1, fig. 1.

Didymocyrtis penultima (Riedel) - Sanfilippo and Riedel, 1980, p. 1010 - Nigrini and Sanfilippo, 2001, p. 241.

Didymocyrtis antepenultima (Riedel and Sanfilippo) (Fig. 3H)

Ommatartus antepenultimus n.sp. Riedel and Sanfilippo, 1970, p. 521, pl. 14, fig. 14.

Didymocyrtis antepenultima (Riedel and Sanfilippo) - Sanfilippo and Riedel, 1980, p. 1010 - Nigrini and Sanfilippo, 2001, p. 233.

Didymocyrtis laticonus (Riedel) (Fig. 3I)

Cannartus laticonus n.sp. Riedel, 1959, p. 291, pl. 1, fig. 5.

Didymocyrtis laticonus (Riedel) - Sanfilippo and Riedel, 1980, p. 1010.

Didymocyrtis antepenultima - Nigrini and Sanfilippo, 2001, p. 237.

Didymocyrtis mammifera (Haeckel) (Fig. 3J)

Cannartidium mammiferum n.sp. Haeckel, 1887, p. 375, pl. 39, fig. 16.

Cannartus mammiferus (Haeckel) - Riedel, 1959, p. 291, pl. 1, fig. 4.

Cannartus mammifer (Haeckel) - Sanfilippo, Burckle, Martini \& Riedel, 1973, p. 216, pl. 1, fig. 7.

Didymocyrtis mammifera (Haeckel)

Sanfilippo and Riedel, 1980, p. 1010 - Nigrini and Sanfilippo, 2001, p. 239.

Didymocyrtis bassanii (Carnevale) (Fig. 3K)

Cannartidium bassanii n.f. Carnevale, 1908, p. 21, pl. 3, fig. 12 .

Didymocyrtis bassanii (Carnevale) - Kamikuri, Motoyama, Nishi \& Iwai, 2009, p. 729, fig. 9H.
Didymocyrtis tetrathalamus (Haeckel) (Fig. 3L)

Panartus tetrathalamus n.sp. Haeckel, 1887, p. 378 , pl. 40 , fig. 3 .

Ommatartus tetrathalamus (Haeckel) - Riedel and Sanfilippo, 1971, p. 1588, pl. 1C, figs. 5-7.

Didymocyrtis tetrathalamus (Haeckel) Sanfilippo and Riedel, 1980, p. 1010.

Didymocyrtis tetrathalamus tetrathalamus (Haeckel) - Nigrini and Sanfilippo, 2001, p. 246.

\section{Genus Saturnalis Haeckel, 1881}

Saturnalis circularis Haeckel (Fig. 3M)

Saturnalis circularis n.sp. Haeckel, 1887, p. 131 - Nigrini, 1967, p. 24, pl. 1, fig. 9.

\section{Genus Druppactractus Haeckel, 1887}

Druppotractus sp. (Fig. 3N)

Remarks: Ellipsoidal shell consisting of a thickwalled external shell and a thinner internal one.

\section{Genus Hexacontium Haeckel, 1881}

Hexacontium armatum/hostile Cleve (Figs. $3 \mathrm{O}$ and $3 \mathrm{P}$ )

Hexacontium armatum n.sp. Cleve, 1900, p. 9, pl. 6, fig. 5 - Boltovskoy and Riedel, 1980, p. 112, pl. 10a-b.

\section{Genus Stylosphaera Ehrenberg, 1846}

Stylosphaera sp. (Fig. 3Q)

Remarks: Actinommid with two prominent three-bladed polar spines.

\section{Genus Plegmosphaera Haeckel, 1887}

Plegmosphaera spp. (Fig. 3R)

Remarks: A central sphere surrounded by an irregularly branched meshwork.

Family PHACODISCIDAE Haeckel, 1881

\section{Genus Heliodiscus Haeckel, 1862}

Heliodiscus spp. (Fig. 3S)

Remarks: Arrangement and abundance of pores quite different from $\mathrm{H}$. asteriscus. Spherical microsphere is laterally located. 
Family COCCODISCIDAE Haeckel, 1862

\section{Genus Periphaena Ehrenberg 1873}

Periphaena decora Ehrenberg (Fig. 3T)

Periphaena decora Ehrenberg, 1873, p. 246; 1875 , pl. 28, fig. 6 - Riedel, 1957, p. 258, pl. 62, fig. 1 - Sanfilippo and Riedel, 1973, p. 523, pl. 8, figs. 8-10; pl. 27, figs. 2-5.

Remarks: Spherical microsphere is apparently located in the centre of the medullary shell.

Family SPONGODISCIDAE Haeckel, 1862

\section{Genus Amphirhopalum Haeckel, 1881}

Amphirhopalum ypsilon Haeckel (Fig. 4A)

Amphirhopalum ypsilon n.sp. Haeckel, 1887, p. 522 - Nigrini and Moore, 1979, p. S75-S77, pl. 10, figs. 1a-e.

\section{Genus Dictyocoryne Ehrenberg, 1860a}

Dictyocoryne truncatum (Ehrenberg) (Fig. 4B)

Rhopalodictyum truncatum n.sp. Ehrenberg, 1861, p. 301.

Dictyocoryne truncatum (Ehrenberg) - Nigrini and Moore, 1979, p. S89, pl. 12, figs. 2a-b.

Dictyocoryne profunda Ehrenberg (Fig. 4C)

Dictyocoryne profunda n.sp. Ehrenberg, 1860a, p. 767 - Nigrini and Moore, 1979, p. S87-S88, pl. 12, fig. 1.

\section{Genus Euchitonia Ehrenberg, 1860a}

Euchitonia furcatalelegans group

(Ehrenberg) (Figs. 4D and 4E)

Pteractis elegans n.sp. Ehrenberg, 1872a, p. 319; 1872b, p. 299, pl. 8, fig. 3.

Euchitonia elegans/furcata group?

(Ehrenberg) - Boltovskoy, 1998, fig. 15.70.

\section{Genus Spongaster Ehrenberg, 1860b}

Spongaster tetras Ehrenberg (Fig. 4F)

Spongaster tetras n.sp. Ehrenberg, 1860b, p. $833 ; 1872$ b, p. 299, pl. 4(3), fig. 8 .

Spongaster tetras tetras Ehrenberg - Nigrini and Moore, 1979, p. S93-S94, pl. 13, fig. 1 Boltovskoy, 1998, fig. 15.67.

Spongaster sp. A aff. D. truncatum (Fig. 4G) aff. Rhopalodictyum truncatum Ehrenberg, 1861, p. 301

Remarks: Test with three spongy arms, having rounded corners

\section{Genus Spongurus Haeckel, 1862}

Spongocore puella Haeckel (Fig. 4H)

Spongocore puella n.sp. Haeckel, 1887, p. 347, pl. 48, fig. 6 - Nigrini and Moore, 1979, p. S59, S61, S69, pl. 8, figs. 5a-c.

Genus Stylodictya Ehrenberg, 1846, 1847, emend. Kozlova in Petrushevskaya and Kozlova, 1972

Stylodictya aculeata Jørgensen (Fig. 4I)

Stylodictya aculeata n.sp. Jørgensen, 1905, pl. 10, fig. 41a-c - Boltovskoy, 1998, fig. 15.62a.

Stylodictya validispina Jørgensen (Fig. 4J)

Stylodictya validispina n.sp, Jørgensen, 1905, p. 119, pl. 10, fig. 40 - Nigrini and Moore, 1979, pl. 13, fig. 5a-b.

\section{Genus Spongodiscus Ehrenberg, 1854}

Spongodiscus sp. (Figs. 4K and 4L)

Remarks: Test a spongy disc, thickened in the centre.

\section{Genus Circodiscus Kozlova in Petrushevskaya} and Kozlova, 1972

Circodiscus spp. (Figs. 4M, 4N, 4O and 4P)

Remarks: circular to sub-circular disc, with several internal concentric rings. Sometimes presents a pylome.

\section{Genus Spongurus Haeckel, 1860}

Spongurus spp. (Figs. 4Q, 4R and 4S)

Remarks: Oval-elongate spongy shell.

\section{Genus Spongopyle Dreyer, 1889}

Spongopyle osculosa Dreyer (Fig. 4T) 
Spongopyle osculosa n.sp. Dreyer, 1889, p. 42, pl. 11, figs. 99-100 - Nigrini and Moore 1979, pl. 15 , fig. 1.

\section{Genus Spongotrochus Haeckel, 1860}

Spongotrochus spp. (Figs. 5A, 5B and 5C)

Remarks: Spongy disc thickened in its central part, sometimes with spines.

Family LITHELIIDAE Haeckel, 1881

\section{Genus Larcopyle Dreyer, 1889}

Larcopyle butschlii Dreyer (Fig. 5D)

Larcopyle bütschlii n. sp. Dreyer, 1889, p. 124, pl. 10,70 - Molina-Cruz, 1977, p. 335, pl. 3, figs. 10-11 - Nigrini and Moore, 1979, p. S131-S132, pl. 17, fig. 1b - Boltovskoy, 1998, figs. 5B-B', 15.85 .

Larcopyle nebulum Lazarus, Faust \& PopovaGoll (Fig. 5E and 5F)

Larcopyle nebulum n.sp. Lazarus, Faust \& Popova-Goll, 2005, p. 111, pl. 5, figs. 1-13.

\section{Genus Lithelius Haeckel 1862}

Lithelius minor Jørgensen (Figs. 5G and 5H)

Lithelius minor n.sp. Jørgensen, 1900, p. 65, pl. 5, fig. 24 - Boltovskoy and Jankilevich, 1985, pl. 3, fig. 17.

\section{Genus Larcospira Haeckel, 1887}

Larcospira spp. (Figs. 5I and 5J)

Remarks: Shell consists of an oval spiral from a common origin.

Larcospira quandrangula Haeckel (Figs. 5K and $5 \mathrm{~L}$ )

Larcospira quadrangula n.sp. Haeckel, 1887, p. 696, pl. 49, fig. 3 - Nigrini and Moore, 1979, p. S133, pl. 17, fig. 2 - Takahashi and Honjo, 1981, p. 150 , pl. 6 , fig. 2 .

Family PYLONIIDAE Haeckel, 1881, emend. Dumitrica, 1989

\section{Genus Phorticium Haeckel, 1881}

Phorticium pylonium group Haeckel (Figs. $5 \mathrm{M}, 5 \mathrm{~N}$ and $5 \mathrm{O}$ )

Phorticium pylonium n.sp. Haeckel, 1887, p. 709, pl. 49, fig. 10.

\section{Genus Pylonium Haeckel, 1881}

Pylonium sp. sensu Benson (Fig. 5P)

Pylonium sp. Benson, 1966, p. 250, pl. 16, fig. 2 - Benson, 1983, p. 507, pl. 6, fig. 5.

\section{Genus Tetrapyle Müller, 1858}

Tetrapyle octacantha group Müller (Fig. 5Q)

Tetrapyle octacantha n.sp. Müller, 1858, p. 33, pl. 2, figs. 12-13; pl. 3, figs. 1-12 - Nigrini and Moore, 1979, p. S125, pl. 16, figs. 3a-b - Takahashi and Honjo, 1981, p. 150, pl. 6, figs. 5-6.

Spumell. gen and sp. indet. (Figs. 5R, 5S and 5T)

Order NASELLARIA Ehrenberg, 1875

Family SPYRIDAE Ehrenberg, 1846

\section{Genus Amphispyris Ehrenberg, 1872b}

Amphispyris reticulata (Ehrenberg) (Fig. 6A)

Dictyospyris reticulata n.sp. Ehrenberg, 1872b, p. 307, pl. 10, fig. 19.

Amphispyris reticulata (Ehrenberg) Boltovskoy, 1998, fig. 15.95 - Tan and Chen, 1999, p. 279, fig. 5-198.

\section{Genus Cantharospyris Haeckel, 1887}

Cantharospyris platybursa Haeckel (Fig. 6B)

Cantharospyris platybursa n.sp. Haeckel, 1887, p. 1051, pl. 53, fig. 7 - Takahashi and Honjo, 1981, p. 152, pl. 7, fig. 32.

\section{Genus Dendrospyris Haeckel, 1881}

Dendrospyris bursa Sanfilippo and Riedel (Fig. 6C)

Dendrospyris bursa n. sp. Sanfilippo and Riedel in Sanfilippo, Burckle, Martini \& Riedel, 1973, p. 217, pl. 2, figs. 9-13 - Nigrini and Lombari, 1984, p. N19, pl. 16, figs. 1a-f Kamikuri, Motoyama, Nishi \& Iwai, 2009, p. 736, fig. 14G. 
Genus Giraffospyris Haeckel, 1887, emend. Goll, 1969

Giraffospyris toxaria (Haeckel) (Fig. 6D)

Podocoronis (Dipocoronis) toxarium n.sp. Haeckel, 1887, p. 980, pl. 83, fig. 7.

Giraffospyris toxaria (Haeckel) - Goll, 1969 , p. 335, pl. 56, figs. 1-2, 4, 7 - Kamikuri, Motoyama, Nishi \& Iwai, 2009, p. 734, fig. 12P.

\section{Genus Liriospyris Haeckel, 1881}

Liriospyris parkerae Riedel and Sanfilippo (Fig. 6E)

Liriospyris parkerae n.sp. Riedel and Sanfilippo, 1971, p. 1590, pl. 2C, fig. 15; pl. 5, fig. 4 - Kamikuri, Motoyama, Nishi \& Iwai, 2009, p. 734 , fig. 12E.

Liriospyris spp. (Figs. 6F, 6G and 6H)

Remarks: Sagittal ring sub-circular; joined directly to front, apex, and back of lattice shell. Vertical spine very short, projecting from approximate midpoint of sagittal ring; no frontal or axial spines.

\section{Genus Lophospyris Haeckel, 1881}

Lophospyris pentagona pentagona

(Ehrenberg) Goll (Fig. 6I)

Ceratospyris pentagona n. sp. Ehrenberg, 1872a, p. 303 , pl. 15 , fig. 5.

Dorcadospyris pentagona (Ehrenberg) Goll, 1969, p. 338-339, pl. 59, figs. 1-3.

Lophospyris pentagona pentagona (Ehrenberg) - Nigrini and Moore, 1979, p. N15-N16, pl. 19, fig. 5 - Takahashi, 1991, p. 102, pl. 28, figs. 9-14.

\section{Genus Neophrospyris Haeckel, 1887}

Nephrospyris renilla Haeckel (Fig. 6J)

Nephrospyris renilla $\mathrm{n}$.sp. Haeckel, 1887, p. 1101, pl. 90, figs. 9-10 - Boltovskoy, 1998, fig. 15.99 .

\section{Genus Androspyris Haeckel, 1887}

Androspyris huxleyi (Haeckel) (Fig. 6K)

Lamprospyris huxleyi n.sp. Haeckel, 1887, pl. 89 , fig. 14 .
Androspyris huxleyi (Haeckel) - Goll, 1980, pl. 4, figs. 4-5.

Genus Zygocircus Butschli 1882, emend. Goll, 1979 Zygocircus productus (Benson) (Fig. 6L)

Zygocircus productus n.sp. Benson, 1966, p. 288, pl. 19, figs. 14-15 - Goll, 1979, p. 381, pl. 2, figs. 4-9.

\section{Genus Phormospyris Goll, 1976}

Phormospyris sp. A (Fig. 6M)

Remarks: Bilocular cephalis, circular to elliptical pores. Sagittal ring asymmetrical, with a line of bigger pores compared to cephalis.

Phormospyris stabilis stabilis (Goll) (Fig. 6N)

Desmospyris anthocyrtoides Benson, 1966, p. 332 , pl. 23 , figs. $6-8$.

Dendrospyris stabilis Goll, 1968, p. 1422, pl. 173, figs. 16-18, 20.

Phormospyris stabilis (Goll) stabilis Goll, 1976, p. 390, pl. 1, figs. 1-13; pl. 2, figs. 7-14 - Takahashi, 1991, p. 104, pl. 30, figs. 2-5 Boltovskoy, 1998, fig. 15.100.

Phormospyris stabilis capoi Goll (Fig. 6O)

Phormospyris stabilis capoi $\mathrm{n}$.sp. Goll, 1976, p. 392, 394, pl. 5, figs. 1-2; pl. 6-7 - Takahashi, 1991, p. 104, pl. 29, figs. 15-18.

Phormospyris stabilis (Goll) scaphipes (Haeckel) (Fig. 6P)

Tristylospiris scaphipes n.sp. Haeckel, 1887, p. 1033, pl. 84, fig. 13 .

Phormospyris stabilis scaphipes (Haeckel) Goll, 1976, p. 394, pl. 8-9.

Tholospyris scaphipes (Haeckel) - MolinaCruz, 1977, p. 336, pl. 7, figs. 11-12.

\section{Genus Tholospyris Haeckel, 1881}

Tholospyris spp. (Figs. 6Q, 6R, 6S and 6T)

Remarks: Oval-shaped sagitally constricted skeleton with very heavy bars and circular to oval pores.

Family PLAGONIIDAE Haeckel, 1881, emend. Riedel, 1957 
Genus Amphiplecta Haeckel, 1887

Amphiplecta acrostoma Haeckel (Fig. 7A)

Amphiplecta acrostoma n.sp. Haeckel, 1887, p. 1223, pl. 97, fig. 10 - Petrushevskaya, 1971, pl. 54, figs. 2-7.

\section{Genus Cladoscenium Haeckel, 1887}

Cladoscenium spp. (Fig. 7B, 7C and 7D)

Remarks: Cephalis large, sub-cylindrical with a rounded or dome-shaped top, with unequal, irregular pores of all shapes. Those specimens present a typical large horn above to the cephalis. The feet are three bladed.

\section{Genus Clathrocanium Ehrenberg, 1860a, emend. Petrushevskaya, 1971}

Clathrocanium sp. (Fig. 7E)

Remarks: Cephalis dome-shaped to globular, with irregular pores. Thorax with three large gates each between adjacent feet; the distal margins of the gates defined by convex downward arched bars developed between adjacent feet. Those specimens present a typical large horn above to the cephalis.

\section{Genus Lophophaena Ehrenberg, 1847}

Lophophaena hispida (Ehrenberg) (Fig. 7F)

Dictyocephalus hispidus n.sp. Ehrenberg 1872b, p. 202, 289, pl. 5, fig. 18.

Lophophaena hispida (Ehrenberg) Boltovskoy, 1998, p. 66, fig. 15.109.

Lophophaena aff. hispida (Fig. 7G)

aff. Dictyocephalus hispidus Ehrenberg 1872b, p. 202, 289; pl. 5, fig. 18

Remarks: Cephalis spherical, with small pores, thin spines. Thorax conical, its pores decreasing in size toward the base.

Lophophaena aff. capito (Fig. 7H)

aff. Lophophaena capito Ehrenberg, 1873, p. 242; 1875, pl.8, fig.6

Remarks: Cephalis spherical, with medium pores, thin spines and a central robust spine in the center of the cephalis.

Plagoniidae group (Figs. 7I, 7J, 7K and 7L) Includes many generally similar forms with a lat- ticed cephalis and with or without a rudimentary thorax. The systematics of these sometimes extremely abundant forms is confused, and they are generally ignored in most surveys. They include species cited under a variety of generic names, such as Amphiplecta, Arachnocorallium, Arachnocorys, Ceratocyrtis, Dimelissa, Lophophaenoma, Micromelissa, Peromelissa, Psilomelissa, etc. (Boltovskoy, 1998).

Pteroscenium spp. (Fig. 7M)

Shell campanulate with irregular circular pores. Horn slender pyramidal. Three divergent feet twice as long as the horn strongly curved, thorny, three-edged.

\section{Genus Pteroscenium Jørgensen, 1905}

Pteroscenium pinnatum Haeckel (Fig. 7N)

Pteroscenium pinnatum n.sp. Haeckel, 1887, pl. 53, figs. 14, 16 - Takahashi, 1991, p. 114, pl. 36, figs. 8-9.

\section{Genus Tetraphormis (Haeckel), 1887}

Tetraphormis dodecaster (Haeckel) (Fig. 7O)

Sethophormis dodecaster n.sp. Haeckel, 1887, p. 1248, pl. 56, fig. 12.

Tetraphormis dodecaster (Haeckel) -

Takahashi, 1991, p. 108, pl. 32, fig. 7.

Clathrocorys aff. teuscheri (Fig. 7P)

aff. Clathrocorys teuscheri Haeckel, 1887, p. 1220, pl. 64, fig. 10

Remarks: it consists of a three-bladed central spine, which bifurcates in two perpendicular spines tree times along the central spine.

\section{Genus Helothus Jørgensen, 1905}

Helothus histricosa Jørgensen (Figs. 7Q and 7R)

Helotholus histricosa Jørgensen, 1905, pl. 16, figs. 86, 88 - Boltovskoy and Riedel, 1987, pl. 4, fig. 4 - Boltovskoy, 1998, 15.113 (a-c).

Helothus aff. histricosa (Fig. 7S)

aff. Helotholus histricosa Jørgensen, 1905, pl. 16 , figs. 86,88

Remarks: Test broad, conical, with generally a broadly rounded, partially hidden cephalis with two robust spines. The pores follow an arrangement compared to $\mathrm{H}$. histricosa. 


\section{Genus Sethophormis Haeckel, 1881}

Sethophormis sp. A (Fig. 7T)

Remarks: Shell in the form of a Chinese peasant hat, thorax with a very delicate lace-like meshwork of irregular pores decreasing in size toward the periphery and many radial sinuous ribs. Shell diameter: $>300 \mu \mathrm{m}$.

Family THEOPERIDAE Haeckel, 1881, emend. Riedel, 1967a in Banner et al., 1967

\section{Genus Artopilium Popofsky, 1913}

Artopilium undulatum Popofsky (Fig. 8A)

Artopilium undulatum n.sp. Popofsky, 1913, p. 405-406, pl. 36, figs. 4-5.

Lophocorys undulata (Popofsky) - Takahashi, 1991, p. 120, pl. 40, figs. 9-10.

\section{Genus Clathtrocyclas Haeckel, 1881}

Clathrocyclas alcmenae Haeckel (Fig. 8B)

Clathrocyclas alcmenae n.sp. Haeckel, 1887, p.1388, pl. 59, fig. 6 .

Clathtrocyclas monumentum (Haeckel) (Fig. 8C)

Calocyclas monumentum n.sp. Haeckel, 1887, p. 138.5, pl. 73, fig. 9 - Takahashi, 1991, p. 112, pl. 34, figs. 9-11.

\section{Genus Corocalyptra Haeckel, 1887}

Corocalyptra kruegeri Popofsky (Figs. 8D, $8 \mathrm{E}$ and $8 \mathrm{~F}$ )

Corocalyptra kruegeri n.sp. Popofsky, 1908, p. 289, pl. 35, fig. 8 - Boltovskoy, 1998, p. 72, fig. 15.136.

\section{Corocalyptra sp. (Fig. 8G)}

Remarks: Cephalis with an apical horn. Thorax large, campanulate, with regular, polygonal pores in transversal rows increasing in size distally. Abdomen restricted to a narrow brim with several rows of pores.

Corocalyptra cervus (Ehrenberg) (Fig. 8H)

Eucyrtidium cervus n.sp. Ehrenberg 1872a, p. 308.

Coracalyptra cervus (Ehrenberg) - Takahashi, 1991, p. 112, pl. 33, figs. 9-12 - Boltovskoy, 1998, p. 71 , fig. 15.128 .

\section{Genus Cornutella Ehrenberg, 1838}

Cornutella profunda Ehrenberg (Fig. 8I)

Cornutella clathrata (?) profunda n.sp.

Ehrenberg, 1854, p. 241.

Cornutella profunda Ehrenberg - Boltovskoy, 1998, p. 71, fig. 15.127 - Takahashi 1991, 113, pl.35, fig.3-9.

\section{Genus Cycladophora Ehrenberg, 1846}

Cycladophora davisiana Ehrenberg (Figs. 8J and $8 \mathrm{~K}$ )

Cycladophora (?) davisiana n.sp. Ehrenberg, 1861, p. 297.

Cycladophora davisiana Ehrenberg - MolinaCruz, 1977, pl. 7, fig. 19.

Theocalyptra davisiana (Ehrenberg) - Nigrini and Moore, 1979, pl. 24, figs. 2a-b.

Cycladophora davisiana Ehrenberg Boltovskoy, 1998, fig. 15.131.

\section{Genus Cyrtocapsella (Haeckel) 1887}

Cyrtocapsella cornuta (Haeckel) (Figs. 8L and 8M)

Cyrtocapsa cornuta n.sp. Haeckel, 1887, p. 1513, pl. 78, fig. 9.

Cyrtocapsella cornuta (Haeckel) - Sanfilippo and Riedel, 1970, p. 453, pl. 1, figs. 19-20 - Kamikuri, Motoyama, Nishi \& Iwai, 2009, p. 731, fig. 10X.

Cyrtocapsella tetrapera (Haeckel) (Figs. 8N and 80 )

Cyrtocapsa tetrapera n.sp. Haeckel, 1887, pl. 78 , fig. 5 .

Cyrtocapsella tetrapera (Haeckel) Sanfilippo and Riedel, 1970, p. 453, pl. 1, figs. 16-18 - Kamikuri, Motoyama, Nishi \& Iwai, 2009, p. 731, fig. $10 \mathrm{Z}$.

Cyrtocapsella cylindroides (Principi) (Fig. 8P)

Sticocapsa cylindroides n.f. Principi, 1909, p. 20, pl. 1, fig. 66 .

Lithocampe (Cyrtocapsella) cylindroides (Principi) - Petrushevskaya, 1975, p. 582, pl. 14, figs. 14-15.

Cyrtocapsella cylindroides (Principi) - Nigrini and Lombari, 1984, p. N103-104, pl. 23, fig. 2.

Cyrtocapsella japonica (Nakaseko) (Figs. $8 \mathrm{Q}, 8 \mathrm{R}$ and $8 \mathrm{~S}$ ) 
Eusyringium japonicum n.sp Nakaseko, 1963, p. 193, pl. 4, figs. 1-3.

Cyrtocapsella japonica (Nakaseko) Sanfilippo and Riedel, 1970, p. 452, pl. 1, figs. 13-15 - Kamikuri, Motoyama, Nishi \& Iwai, 2009, p. 731, fig. 10Y.

\section{Cyrtocapsella sp. (Fig. 8T)}

Remarks: Cephalis is spherical, cylindrical shape in general. Thorax is the same size of abdomen and post-abodominal chamber. The surface is rough. Pores are round and the approximately the same size in the entire specimen. $\sim 150 \mu \mathrm{m}$ of length.

\section{Genus Dictyophimus Ehrenberg, 1842, emend. Nigrini, 1968} and 9B)

Dictyophimus infabricatus Nigrini (Figs. 9A

Dictyophimus infabricatus n.sp. Nigrini, 1968, p. 56, pl. 1, fig. 6 - Takahashi, 1991, pl. 37, figs. 3-5.

Dictyophimus hirundo (Haeckel) (Fig. 9C)

Pterocorys hirundo n.sp Haeckel, 1887, p. 1318, pl. 71, fig. 4.

Dictyophimus hirundo group (Haeckel) Nigrini and Moore, 1979, pl. 22, figs. 2-4.

Dictyophimus spp. (Figs. 9D, 9E and 9F)

Remarks: Cephalis partly submerged into thorax, bears a large apical horn. Thorax pyramidal or conical, with three conspicuous legs.

\section{Genus Eucecryphalus Haeckel, 1860}

Eucecryphalus gegenbauri Haeckel (Fig. 9G)

Eucecryphalus gegenbauri n.sp. Haeckel, 1860, p. 836 - Haeckel, 1862, p. 308, pl. 5, figs. 12-15.

Theocalyptra gegenbauri (Haeckel) Boltovskoy and Riedel, 1980, p. 126, pl. 5, fig. 18.

Eucecryphalus tricostatum (Haeckel) (Figs. 9H, 9I, 9J and 9K)

Theopilium tricostatum n.sp. Haeckel, 1887, p. 1322, pl. 70, fig. 6 - Takahashi and Honjo,
1981, p. 152, pl. 8, fig. 12 - Boltovskoy, 1998, p. 78 , fig. 15.149 .

Eucecryphalus spp. (Figs. 9L, 9M, 9N, 9O, 9P, 9Q, 9R, 9S and 9T)

Remarks: Shell with a small cephalis. Thorax widely open, with small, regularly arranged pores, circular proximally and becoming larger and more polygonal distally. Abdominal brim flat with pores. In some specimens the abdominal brim is not observe because is broken.

\section{Genus Eucyrtidium Ehrenberg, 1847, emend. Nigrini, 1967}

Eucyrtidium anomalum Haeckel (Fig. 10A)

Eucyrtidium anomalum n.sp. Haeckel, 1862, p. 323, pl. 7, figs. 11-13 - Boltovskoy and Riedel, 1987, p. 100, pl. 5, fig. 1 - Boltovskoy, 1998, p. 75 , fig. 15.138 .

Eucyrtidium calvertense Martin (Fig. 10B)

Eucyrtidium calvertense n.sp. Martin, 1904, p. 450-451, pl. CXXX, fig. 5 - Chen, 1975, p. 460, pl. 15 , fig. 9.

Eucyrtidium cienkowskii Haeckel (Figs. 10C and 9D)

Eucyrtidium cienkowskii n.sp. Haeckel, 1887, pl. 80, fig. 9 - Sanfilippo, Burckle, Martini \& Riedel, 1973, p. 221, pl. 5, figs. 7-11 - Lazarus, 1990, pl. 6, figs. 1-3.

Eucyrtidium hexagonatum Haeckel (Fig. $10 \mathrm{E}, 10 \mathrm{~F}$ and $10 \mathrm{G}$ )

Eucyrtidium hexagonatum n.sp. Haeckel, 1887, p. 1489, pl. 80, fig. 11 - Molina-Cruz, 1977, p. 336, pl. 7, figs. 4-5 - Takahashi, 1991, p. 124, pl. 42, figs. 18-19.

Eucyrtidium hexastichus (Haeckel) (Fig. 10H)

Lithostrobus hexastichus n.sp. Haeckel, 1887, p. 1470, pl. 80, fig. 15.

Eucyrtidium hexastichum (Haeckel) Boltovskoy and Riedel, 1980, p. 124, pl. 5, fig. 10 - Takahashi, 1991, p. 125, pl. 42, fig. 22 Boltovskoy, 1998, p. 75, fig. 15.139. 
Eucyrtidium teuscheri Haeckel (Fig. 10I)

Eucyrtidium teuscheri n.sp. Haeckel, 1887, p. 1491, pl. 77, fig. 5 - Caulet, 1986, pl. 5, figs. 1-8.

Eucyrtidium spp. (Figs. 10J, 10K, 10L, 10M, $10 \mathrm{~N}, 10 \mathrm{O}, 10 \mathrm{P}, 10 \mathrm{Q}, 10 \mathrm{R}, 10 \mathrm{~S}$ and 10T)

Remarks: Small spherical to sub-spherical cephalis with small apical horn. Thorax small, inflated, thick-walled. Abdomen and three-five post-abdominal segments thin-walled, with pores arranged in longitudinal rows.

\section{Genus Lampromitra Haeckel, 1887}

Lampromitra schultzei Haeckel (Fig. 11A)

Lampromitra schultzei n.sp. Haeckel, 1887, p. 1214, pl. 60, figs. 7-7a. - Boltovskoy, 1998, p. 63, pl. 15.115 .

\section{Genus Litharachnium Haeckel, 1860}

Litharachnium tentorium Haeckel (Fig. 11B)

Litharachnium tentorium n.sp. Haeckel, 1860 , p. 836 ; 1862 , p. 281 , pl. 4, figs. $7-10-$ Boltovskoy, 1998, p. 75, fig. 15.148.

\section{Genus Lipmanella Loeblich and Tappan, 1961}

Lipmanella dictyoceras (Haeckel) (Figs. 11C and 11D)

Lithornithium dictyoceras Haeckel, 1860, p. 840.

Lipmanella virchowii Boltovskoy and Riedel, 1987, p. 100, pl. 5, fig. 6 .

Lipmanella dictyoceras Takahashi, 1991, p. 121, pl. 40, fig. 17 - Boltovskoy, 1998, fig. 15.134.

\section{Lipmanella sp. (Fig. 11E)}

Remarks: Cephalis large, hemispherical, with a conspicuous apical horn. Thorax thin-walled, conical-inflated, in this specimen the thorax is smaller than L. dictyoceras.

\section{Genus Lithopera Ehrenberg, 1846}

Lithopera neotera Sanfilippo and Riedel (Figs. 11F, 11G and 11H)

Lithopera neotera n.sp. Sanfilippo and Riedel, 1970, pl. 1, figs. 24-26 - Kamikuri, Motoyama, Nishi \& Iwai, 2009, p. 731, fig. 10AC.
Lithopera renzae Sanfilippo and Riedel (Figs. 11I, 11J, 11K and 11L)

Lithopera renzae n.sp. Sanfilippo and Riedel, 1970, p. 454, pl. 1, figs. 21-23, 27 - Kamikuri, Motoyama, Nishi \& Iwai, 2009, p. 731, fig. 10T.

Lithopera thornburgi Sanfilippo and Riedel (Fig. 11M)

Lithopera thornburgi n.sp. Sanfilippo and Riedel, 1970, p. 455, pl. 2, figs. 4-6 - Kamikuri, Motoyama, Nishi \& Iwai, 2009, p. 731, fig. 10 AE.

\section{Genus Lithostrobus Haeckel, 1887}

Lithostrobus hexagonalis Haeckel (Fig. 11N)

Lithostrobus hexagonalis n.sp. Haeckel, 1887, p. 1475, pl. 79, fig. 20 - Boltovskoy and Riedel, 1987, p. 100; pl. 5, fig. 11 - Takahashi, 1991, p. 122, pl. 41, figs. 1-3 - Boltovskoy, 1998, fig. 15.141.

\section{Genus Lophocyrtis Haeckel, 1881}

Lophocyrtis brachythorax (Sanfilippo and Riedel) (Fig. 11O)

Cyclampterium (?) brachythorax n.sp. Sanfilippo and Riedel, 1970, pl. 2, figs. 15-16.

Lophocyrtis (Cyclampterium) brachythorax Sanfilippo and Riedel, 1970, p. 457, pl. 2, figs. 1516 - Sanfilippo, 1990, pl. IV, figs. 4-6 - Kamikuri, Motoyama, Nishi \& Iwai, 2009, p. 731, fig. 13D.

Lophocyrtis tanythorax Sanfilippo and Riedel (Fig. 11P)

Cyclampterium (?) tanythorax n.sp. Sanfilippo and Riedel, 1970, pl. 2, figs. 13-14.

Lophocyrtis (Cyclampterium) tanythorax Sanfilippo and Riedel, 1970, p. 457, pl. 2, figs. 13-14 - Sanfilippo, 1990, pl. 4, figs. 7-10.

Lophocyrtis spp. (Figs. 11Q and 11R)

Remarks: Shell robust, sub-spherical to subellipsoidal in general form. Cephalis sub-spherical, poreless or with a few small pores, with a short apical horn. Thorax inflated, with pores arranged in groups of 8-10, surrounded by a bigger pore. Abdomen thick-walled, with sub-circular pores, it is slightly convex to inflated. 
Nassell. gen. and sp. indet. (Figs. 11S and 11T)

\section{Genus Pterocanium Ehrenberg, 1846}

Pterocanium grandiporus (Figs. 12A and 12B)

Pterocanium grandiporus n.sp. Nigrini, 1968, p. 57, pl. 1, fig. 7.

Pterocanium praetextum (Haeckel) (Figs. $12 \mathrm{C}$ and 12D)

Lychnocanium praetextum n.sp. Ehrenberg, 1872b, p. 297, pl. 10, fig. 2.

Pterocanium praetextum praetextum (Ehrenberg) - Nigrini and Moore, 1979, p. N41-N42, pl. 23, fig. 2 - Takahashi, 1991, p. 115, pl. 36, figs. 15-18.

Pterocanium praetextum group? (Ehrenberg) - Boltovskoy, 1998, fig. 15.146.

Pterocanium trilobum (Haeckel) (Figs. 12E, $12 \mathrm{~F}, 12 \mathrm{G}$ and $12 \mathrm{H})$

Dictyopodium trilobum n.sp. Haeckel, 1860, p. 839.

Dictyopodium trilobum Haeckel, 1862, p. 340, pl. 8, figs. 6-10.

Pterocanium trilobum (Haeckel) - Nigrini and Moore, 1979, p. N45-N46, pl. 23, figs. 4a-c Boltovskoy, 1998, p. 78, fig. 15. 145.

\section{Genus Stichocorys Haeckel, 1881}

Stichocorys armata (Haeckel) (Figs. 12I and 12J)

Cyrtophormis armata n.sp. Haeckel, 1887, p. 1460, pl. 78, fig. 17.

Stichocorys armata (Haeckel) - Riedel and Sanfilippo, 1971, p. 1595, pl. 2E, figs. 13-15-Kamikuri, Motoyama, Nishi \& Iwai, 2009, p. 731, fig. 100.

Stichocorys delmontensis (Campbell and Clark) (Figs. 12K and 12L)

Eucyrtidium delmontense n.sp. Campbell and Clark, 1944, p. 56, pl. 7, figs. 19-20.

Stichocorys delmontensis Campbell and Clark - Sanfilippo and Riedel, 1970, p. 451, pl. 1, fig. 9 - Kamikuri, Motoyama, Nishi \& Iwai, 2009, p. 731 , fig. 10Q.

Stichocorys peregrina (Riedel) (Fig. 12M)

Eucyrtidium elongatum peregrinum Riedel, 1953, p. 812, pl. 85, fig. 2.
Stichocorys peregrina Riedel - Riedel and Sanfilippo, 1970, p. 451, pl. 1, fig. 10 Foreman, 1975, p. 622, pl. 9, figs. 1-4 - Kamikuri, Motoyama, Nishi \& Iwai, 2009, p. 731, fig. 10P.

Stichocorys sp. (Fig. 12N)

Remarks: Similar to S. peregrina, but the second segment is longer instead to be the third.

\section{Genus Stichopilium Haeckel, 1881}

Stichopilium bicorne (Figs. $12 \mathrm{O}$ and 12P)

Stichopilium bicorne n.sp. Haeckel, 1887, p. 1437, pl. 77, fig. 9 - Nigrini and Moore, 1979, p. N91, pl. 26, figs. 1a-b - Boltovskoy, 1998, p. 83, fig. 15.161.

\section{Genus Theocorys Haeckel, 1881}

Theocorys (?) spp. (Figs. 12Q, 12R and 12S)

Remarks: Test relatively small, consisting of a cephalis, thorax, and abdomen. Apical robust horn. Pores are distributed through the shell irregularly and dispersed.

Theocorys veneris Haeckel (Fig. 12T)

Theocorys veneris Haeckel, 1887 p. 1415, pl. 69, fig. 5 - Boltovskoy and Riedel, 1987, p. 100, pl. 5, fig. 19 - Takahashi, 1991, p. 120, pl. 40, figs. $11-14$

\section{Genus Carpocanarium Haeckel, 1887, emend.} Nigrini and Moore, 1979

Carpocanarium papillosum (Ehrenberg)

(Figs. 13A and 13B)

Eucyrtidium papillosum Ehrenberg, 1872a, p. 310-311.

Carpocanarium papillosum (Ehrenberg) Takahashi, 1991, p. 132, pl. 45, figs. 16-17.

Dictyocephalus papillosus (Ehrenberg) - van de Paverd, 1995, p. 255, pl. 76, fig. 24 Boltovskoy, 1998, figs. 15.135 a-c.

Carpocanarium aff. papillosum (Figs. 13C and 13D)

aff. Eucyrtidium papillosum Ehrenberg, 1872a, p. 310-311

Remarks: Similar to this species, but rearrangement of the pores and peristome is different. 
Family CARPOCANIIDAE Haeckel, 1881, emend. Riedel, 1971a

Genus Carpocanistrum Haeckel, 1887 Carpocanistrum acutidentatum Takahashi (Figs. 13E and 13F)

Carpocanistrum acutidentatum n.sp. Takahashi, 1991, p. 132, pl. 45, figs. 9, 13-15.

Carpocanistrum spp. (Figs. 13G, 13H and 13I) Remarks: Shell outline sub-spherical to oval. Cephalis indistinguishable from thorax, included within its upper part. Peristome poreless, smooth or provided with teeth. Height of shell: $80-130$ $\mu \mathrm{m}$. (Boltovskoy, 1998)

\section{Genus Carpocanopsis Riedel and Sanfilippo, 1971} Carpocanopsis cristata (Carnevale) (Figs. $13 \mathrm{~J}$ and $13 \mathrm{~K}$ )

Sethocorys cristata n.sp. Carnevale, 1908, p. 31, pl. 4, fig. 18.

Carpocanopsis cristatum (Carnevale) Riedel and Sanfilippo, 1971, p. 1597, pl. 1G, fig. 16, pl. 2G, figs. 1-7.

Carpocanopsis cristata (Carnevale) Sanfilippo and Riedel, 1973, p. 531.

Family CANNOBOTRYIDAE Haeckel, 1881

\section{Genus Botryocyrtis Ehrenberg, 1860a, b}

Botryocyrtis scutum (Harting) (Figs. 13L, $13 \mathrm{M}$ and $13 \mathrm{~N}$ )

Haliomma scutum n.sp. Harting, 1863, p. 11, pl. 1, fig. 18 - Molina-Cruz, 1977, p. 338, pl. 6, fig. 14 - Takahashi, 1991, p. 135, pl. 46, figs. 6-7 - Boltovskoy, 1998, p. 88, fig. 15. 172.

Botryocyrtis sp. A (Fig. 130)

Remarks: Eucephalic chamber is enclosed in a rounded cephalis shape. Cylindrical thorax with very small pores. Shell height: $\sim 50 \mu \mathrm{m}$. Present two large feelers.

Genus Botryopyle Haeckel, 1881, emend. Petrushevskaya, 1965

Botryopyle dictyocephalus Haeckel (Figs. 13P and 13Q)
Botryopyle dictyocephalus Haeckel, 1887, p. 1113, pl. 96, fig. 6 .

Botryopyle spp. (Figs. 13R and 13S)

Remarks: Large cephalis, from ovoid to pointed shape, thin-walled. Thorax cylindrical, with pores or poreless tube.

\section{Genus Centrobotrys Petrushevskaya, 1965}

Centrobotrys thermophila Petrushevskaya (Fig. 13T)

Centrobotrys thermophila Petrushevskaya, 1965, p. 115, fig. 20 - Nigrini, 1967, p. 49, fig. 26, pl. 5, fig. 7 - Boltovskoy, 1998, p. 88, fig. 15. 175.

Family ARTOSTROBIIDAE Riedel, 1967a in Banner et al., 1967 and Riedel, 1967b, emend. Foreman, 1973

\section{Genus Artostrobus Haeckel, 1887}

Artostrobus annulatus (Bailey) (Fig. 14A)

Cornutella (?) annulata Bailey, 1856, p. 3, pl. 1, figs. 5a-b.

Artostrobus annulatus (Bailey) - Molina-Cruz, 1991, fig. 2(12-13) - Boltovskoy, 1998, fig. 15.125.

\section{Genus Botryostrobus Haeckel, 1887}

Botryostrobus aquilonaris (Bailey) (Figs. $14 \mathrm{~B}$ and $14 \mathrm{C})$

Eucyrtidium aquilonaris n.sp. Bailey, 1856, p. 4 , pl. 1 , fig. 9 .

Botryostrobus aquilonaris (Bailey) - Nigrini, 1977, p. 246, pl. 1, fig. 1 - Takahashi, 1991, p. 128, pl. 44, figs. 9-13 - Boltovskoy, 1998, p. 84, fig. 15.163.

Botryostrobus auritus (Ehrenberg) (Figs. 14D and 14E)

Lithocampe aurita n.sp. Ehrenberg, 1844, p. 84. Artostrobium auritum group (Ehrenberg) - Riedel and Sanfilippo, 1971, p. 1599, pl. 1H, figs. 5-8.

Lithostrobus lithobotrys (Ehrenberg) Molina-Cruz, 1977, p. 336, pl. 7, fig. 17.

Botryostrobus auritus/australis - Boltovskoy, 1998, p. 84, fig. 15.168. 
Botryostrobus bramlettei (Campbell and Clark) (Fig. 14F)

Lithomitra (Lithomitrissa) bramlettei n.sp. Campbell and Clark, 1944, p. 53, pl. 7, figs. 10-14.

Botryostrobus bramlettei (Campbell and Clark) - Nigrini, 1977, p. 248, pl. 1, figs. 7-8 Kamikuri, Motoyama, Nishi \& Iwai, 2009, p. 730, fig. $10 \mathrm{G}$.

Remarks: difference from B. aquilonaris by having a conspicuously swollen third joint, one of important distinguishing characters of species; cephalis small, rather smooth, caplike, not much pitted, apically rounded, and laterally convex.

\section{Genus Phormostichoartus Campbell, 1951, emend. Nigrini, 1977}

Phormostichoartus corbula (Harting) (Figs. $14 \mathrm{G}$ and $14 \mathrm{H})$

Lithocampe corbula n.sp. Harting, 1863, p. 12, pl. 1, fig. 21.

Phormostichoartus corbula (Harting) Takahashi and Honjo, 1981, p. 154, pl. 10, figs. 13-14 - Boltovskoy and Riedel, 1987, p. 101, pl. 6, fig. 7 - Takahashi, 1991, p. 129, pl. 44, figs. 1416 - Boltovskoy, 1998, p. 84, fig. 15.163.

Phormostichoartus doliolum (Riedel and Sanfilippo) (Fig. 14I)

Artostrobium doliolum n.sp. Riedel and Sanfilippo, 1971, p. 1599, pl. 1H, figs. 1-3; pl. 8, figs. 14-15.

Phormostichoartus doliolum (Riedel and Sanfilippo) - Nigrini, 1977, pl. 1, fig. 14 - Nigrini and Lombari, 1984, p. N181, pl. 31, fig. 5a-b.

Phormostichoartus marylandicus (Martin) (Fig. 14J)

Lithocampe marylandica n.sp. Martin, 1904, p. 450 , pl. 130, fig. 4 .

Artostrobium sp. aff. A. doliolum - Riedel and Sanfilippo, 1971, pl. 1H, fig. 4; pl. 21, figs. 1-8; pl. 3E, figs. 7-9.

Phormostichoartus marylandicus (Martin) Nigrini, 1977, p. 253, pl. 2, figs. 1-3.

\section{Genus Siphocampe Haeckel, 1881, emend.} Nigrini, 1977

Siphocampe spp. A (Figs. 14K and 14L)

Remarks: Specimens present pore rows in the extended part of the abdomen. 14M)

Siphocampe arachnea (Ehrenberg) (Fig.

Lithocampe lineata n.sp. Ehrenberg, 1838, p. 130.

Eucyrtidum lineatum arachneum Ehrenberg, 1861, p. 299.

Lithomitra lineata group (Ehrenberg) Riedel and Sanfilippo, 1971, p. 1600, pl. 1I, figs. 1, 3-11; pl. 21, figs. 14-16.

Siphocampe arachnea (Ehrenberg) - Nigrini, 1977, p. 255, pl. 3, figs. 7-8.

Siphocampe lineata (Ehrenberg) (Fig. 14N)

Lithocampe lineata n.sp. Ehrenberg, 1838, p. 130.

Siphocampe lineata (Ehrenberg) - Nigrini, 1977, p. 256, pl. 3, fig. 9-10.

\section{Genus Siphostichoartus Nigrini, 1977}

Siphostichoartus corona (Haeckel) (Figs. $14 \mathrm{O}$ and $14 \mathrm{P}$ )

Cyrtophormis (Acanthocyrtis) corona n.sp. Haeckel, 1887, p. 1462. pl. 77, fig. 15.

Phormostichoartus corona (Haeckel) Riedel and Sanfilippo, 1971, p. 1600, pl. 1I, figs. 13-15; pl. 2J, figs. 1-5.

Siphostichoartus corona (Haeckel) - Nigrini, 1977, p. 257, pl. 2, figs. 5-6.

\section{Genus Spirocyrtis Haeckel, 1881, emend.} Nigrini, 1977

Spirocyrtis gyroscalaris Nigrini (Fig. 14Q)

Spirocyrtis gyroscalaris n.sp. Nigrini, 1977, pl. 2, figs. 10-11.

Spirocyrtis gyroscalaris Nigrini - Kamikuri, Motoyama, Nishi \& Iwai, 2009, p. 730, fig. 10E.

Spirocyrtis scalaris Haeckel (Figs. 14R and 14S) 
Spirocyrtis scalaris n.sp. Haeckel, 1887, p. 1509, pl. 76, fig. 14 - Nigrini, 1977, p. 259, pl. 2 , figs. 12-13 - Takahashi, 1991, p. 127, pl. 44, figs. 1-2 - Boltovskoy, 1998, p. 85, fig. 15.166.

Spirocyrtis subtilis Petrushevskaya (Fig. 14T) Spirocyrtis subtilis n.sp. Petrushevskaya in Petrushevskaya and Kozlova, 1972, p. 540, pl. 24, figs. 22-24 - Nigrini, 1977, p. 260, pl. 3, fig. 3 - Kamikuri, Motoyama, Nishi \& Iwai, 2009, p. 730, fig. 10L.

Family PTEROCORYTHIDAE Haeckel, 1881, emend. Riedel, 1967a in Banner et al., 1967, emend. Moore, 1972

Pterocorida Haeckel, 1881, p. 435

Pterocoryidae Riedel, 1967a in Banner et al., 1967, p. 296

\section{Genus Anthocyrtidium Haeckel, 1881}

Anthocyrtidium ehrenbergi (Stöhr) (Figs.

15A and 15B)

Anthocyrtis ehrenbergi n.sp. Stöhr, 1880, p. 100, pl. 3, fig. 21a-b.

Anthocyrtidium ehrenbergi (Stöhr) - Nigrini and Lombari, 1984, N147, pl. 27, fig. 1.

Anthocyrtidium ophirense (Ehrenberg) (Figs. $15 \mathrm{C}$ and $15 \mathrm{D})$

Anthocyrtis ophirensis n.sp. Ehrenberg, 1872 b, p. 285, pl. 9, fig. 13.

Anthocyrtidium ophirense (Ehrenberg) - MolinaCruz, 1977, p. 337, pl. 6, fig. 10 - Nigrini and Moore, 1979, p. N67, pl. 25, fig. 1 - Takahashi, 1991, p. 126, pl. 43, figs. 1-7 - Boltovskoy, 1998, fig. 15.152.

Anthocyrtidium spp. (Figs. 15E and 15F)

Remarks: Several variations in these specimens. In general consists of a cephalis elongate with a robust three-bladed apical horn. Thorax campanulate, with circular, regular pores arranged longitudinally, peristome distinct within small teeth or undulate. Abdomen is absent.

\section{Genus Calocycletta Haeckel, 1887}

Calocycletta caepa Moore (Figs. 15G and 15H)

Calocycletta caepa n.sp. Moore, 1972, p. 150, pl. 2, figs. 4-7 - Kamikuri, Motoyama, Nishi \& Iwai, 2009, p. 736, fig. 14E.

Calocycletta virginis Haeckel (Fig. 15I)

Calocyclas (Calocycletta) virginis n.sp. Haeckel, 1887, p. 1381, pl. 74, fig. 4.

Calocycletta virginis Haeckel - Riedel and Sanfilippo, 1978, p. 66, pl. 3, figs. 13-14.

\section{Genus Lamprocyclas Haeckel, 1887, emend.} Nigrini, 1967

Lamprocyclas junonis (Haeckel) (Fig. 15J)

Theoconus junonis n.sp. Haeckel, 1887, p. 1401, pl. 69, fig. 7.

Lamprocyclas junonis (Haeckel) - Caulet, 1986, pl. 4, fig. 10.

Lamprocyclas maritalis group Haeckel (Figs. $15 \mathrm{~K}$ and $15 \mathrm{~L}$ )

Lamprocyclas maritalis n.sp. Haeckel, 1887, p. 1390, pl. 74, figs. 13-14.

Lamprocyclas maritalis maritalis Haeckel Nigrini, 1967, p. 74-76, pl. 7, fig. 5 - Molina-Cruz, 1977, p. 337, pl. 7, figs. 8-9 - Nigrini and Moore, 1979, p. N75, pl. 25, fig. 4 - Boltovskoy, 1998, fig. 15.158.

\section{Lamprocyclas sp. A (Fig. 15M)}

Remarks: Cephalis sub-spherical, elongate, with a large three-bladed apical horn. Thorax campanulate, thick-walled, with sub-regular, circular pores. Abdomen, with large, sub-regular, circular pores and with sub-terminal teeth.

\section{Genus Lamprocyrtis Kling, 1973}

Lamprocyrtis nigriniae (Caulet) (Fig. 15N)

Conarachnium nigriniae n.sp. Caulet, 1971, p. 3, pl. 3, figs. 1-4; pl. 4, figs. 1-4.

Lamprocyrtis nigriniae (Caulet) - Nigrini and Moore, 1979, p. N81-N82, pl. 25, fig. 7 - Takahashi, 1991, p. 127, pl. 43, figs. 17-19 Boltovskoy, 1998, p. 82, fig. 15.157. 
Lamprocyrtis spp. (Figs. $15 \mathrm{O}$ and 15P)

Remarks: Cephalis sub-spherical, with a large three bladed horn. Thorax campanulate, thinwalled, with large polygonal pores increasing in size distally. No abdomen.

\section{Genus Pterocorys Haeckel, 1881, emend. Pe- trushevskaya, 1971}

Pterocorys minythorax (Nigrini) (Fig. 15Q)

Theoconus minythorax n.sp. Nigrini, 1968, p. 57, pl. 1, fig. 8 - Molina-Cruz, 1977, p. 338, pl. 7, fig. 1.

Pterocorys minythorax (Nigrini) - Nigrini and Moore, 1979, p. N87, pl. 25, fig. 10 - Boltovskoy, 1998 , p. 82 , fig. 15.156 .

Pterocorys zancleus (Müller) (Figs. 15R and 15S) Eucyrtidium zancleum n.sp. Müller, 1855, p. 672.

Pterocorys zancleus (Müller) - Boltovskoy, 1998, p. 82, fig. 15.159 - Kamikuri, Motoyama, Nishi \& Iwai, 2009, p. 732, fig. 11R.

Pterocorys sp. (Fig. 15T)

Remarks: Not well preserved specimen.

\section{ACKNOWLEGMENT}

The Swiss IODP committee is acknowledged for giving us the opportunity to participate in this project. Shipboard data and samples were provided by the Integrated Ocean Drilling Program (IODP). Special thanks to Dr. Demetrio Boltovskoy and Dr. Valentin Chesnel for their the revi-sion and support on this work. The Swiss National Science Foundation (SNSF) financially supported this research through projects 200021134873 and 200020-143894.

\section{REFERENCES}

Bailey, J. W. (1856). Notice of microscopic forms found in the soundings of the Sea of Kamtschatka. In B. Silliman, B. Silliman Jr. \& J. D. Dana (Eds.), The American Journal of Science and Arts, 22, 1-6 (+ 1 plate).
Banner, F. T., Clarke, W. J., Cutbill, J. L., Eames, F. E., Lloyd, A. J., Riedel, W. R. \& Smout, A. H. (1967). Chapter 8 Protozoa. In W. B. Harland, C. H. Holland, M. R. House, N. F. Hughes, A. B. Reynolds, M. J. S. Rudwick, ... E. C. Wiley (Eds.), The Fossil Record (Vol. 2, pp. 291-332). doi: 10.1144/GSL. SP.1967.002.01.26

Benson, R. N. (1966). Recent Radiolaria from the Gulf of California (Unpublished doctoral dissertation). Minnesota University, Minnesota, United States of America.

Benson, R. N. (1983). Quaternary Radiolarians from the Mouth of the Gulf of California, Leg 65 of the Deep Sea Drilling Project. Reports of the Deep Sea Drilling Project, 65, 491-523. doi: 10.2973/dsdp. proc.65.123.1983

Bjørklund, K. R., Cortesea, G., Swanberg, N. \& Schrader, H. J. (1998). Radiolarian faunal provinces in surface sediments of the Greenland, Iceland and Norwegian (GIN) Seas. Marine Micropaleontology, 35(1-2), 105-140. doi: 10.1016/S03778398(98)00013-9

Boltovskoy, D. (1994). The sedimentary record of pelagic biogeography. Progress in Oceanography, 34(2-3), 135-160. https:// doi.org/10.1016/0079-6611(94)90006-X

Boltovskoy, D. (1998). Classification and distribution of South Atlantic recent Polycystine radiolaria. Palaeontologia Electronica, 1(2;6A), 111 pp.

Boltovskoy, D. (1999). Radiolaria Polycystina. In D. Boltovskoy (Ed.), South Atlantic Zooplankton (pp. 149-212). The Netherlands: Backhuys Publishers.

Boltovskoy, D. \& Jankilevich, S. S. (1985). Radiolarian distribution in East equatorial Pacific plankton. Oceanologica Acta, 8(1), 101-123. 
Boltovskoy, D. \& Riedel, W. R.(1980). Polycystine Radiolaria from the Southwestern Atlantic Ocean plankton. Revista Española de Micropaleontología, 12(1), 99-146.

Boltovskoy, D. \& Riedel, W. R.(1987). Polycystine radiolaria of the California Current region: Seasonal and geographic patterns. Marine Micropaleontology, 12(1), 65-104. doi: 10.1016/0377-8398(87)90014-4

Brandt, K. (1905). Zur Systematik der koloniebildenden Radiolarien. Zoologische Jahrbücher. Supplement, VIII, 311-352.

Bütschli, O. (1882). Beiträge zur Kenntnis der Radiolarienskelette, insbesondere der der Cyrtida. Zeitschrift für Wissenschaftliche Zoologie, 36, 485-540.

Cachon, J. \& Cachon, M. (1972a). Le système axopodial des Radiolaires Sphaeroidés I. Centroaxoplastidiés [The axopodial system of radiolaria Sphaeroidae I. Centroaxoplastididae]. Archiv Für Protistenkunde, 114, 51-64.

Cachon, J. \& Cachon, M. (1972b). Le système axopodial des Radiolaires Sphaeroïdés II. Les Periaxoplastidiés III. Les cryptoaxoplastidiés (anaxoplastidiés) IV. Les fusules et le système rhéoplasmique. Archiv Für Protistenkunde, 114, 291-307.

Calkins, G. N. (1909). Protozoölogy (Lea \& Febiger). New York and Philadelphia.

Campbell, A. S. (1951). New genera and subgenera of Radiolaria. Journal of Paleontology, 25(4), 527-530.

Campbell, A. S. \& Clarck, B. L. (1944). Miocene radiolarian faunas from southern California. Geological Society of America, Special Papers, 51, 1-76.

Carnevale, P. (1908). Radiolarie e Silicoflagellati di Bergonzano (Reggio Emilia). Estrato dalle Memorie del Reale Istituto Veneto di Scienze, Lettere ed Arti, XXVIII(3), 1-46.

Caulet, J.-P. (1971). Contribution à l'étude de quelques Radiolaires Nassellaires des boues de la Méditerranée et du Pacifique. Cahiers de Micropaleontologie, 2(10), 1-10.

Caulet, J.-P. (1986). 14. Radiolarians from the Southwest Pacific. In R. B. Blakeslee (Ed.), Initial Reports of the Deep Sea Drilling Project (Vol. 90, pp. 835-861). https://doi. org/10.2973/dsdp.proc.90.114.1986

Chen, M. \& Tan, Z. (1989). Description of a new genus and 12 new species of Radiolaria in sediments from the South China Sea. Tropic Oceanology, 8(1), 1-9.

Chen, P.-H. (1975). 11. Antarctic Radiolaria. Initial Reports of the Deep Sea Drilling Project, 28, 437-513. doi: 10.2973/dsdp. proc.28.111.1975

Cleve, P. T. (1900). Notes on some Atlantic Plankton-organisms. Kongliga Svenska Vetenskaps-Akademiens Handlingar, 34(1), 1-22 + plates.

De Wever, P., Dumitrica, P., Caulet, J. P., Nigrini, C. \& Caridroit, M. (2001). Radiolarians in the Sedimentary Record. Amsterdam: Gordon and Breach Science Publishers.

Dreyer, F. (1889). Die Pylombildungen in vergleichend-anatomischer und entwicklungsgeschichtlicher Beziehung bei Radiolarien und bei Protisten uberhaupt, nebst System und Beschreibung neuer und der bis jetzt bekannten pylomatischen Spumellarien. Jenaische Zeitschrift Für Naturwissenschaft, 23, 1-138 + tables.

Dumitrica, P. (1989). Internal skeletal structures of the superfamily Pyloniacea [Radiolaria], a basis of a new systematics. Revista Española de Micropaleontología, 21(2), 207-264. 
Ehrenberg, C. G. (1838). Über die Bildung der Kreidefelsen und des Kreidemergels durch unsichtbare Organismen. Abhandlungen der Königlichen Akademie der Wissenschaften zu Berlin, 59-147 + tables and plates.

Ehrenberg, C. G. (1844). Über 2 neue Lager von Gebirgsmassen aus Infusorien als Meeres-Absatz in Nord-Amerika und eine Vergleichung derselben mit den organischen Kreide-Gebilden in Europa und Afrika. Bericht Über Die Zur Bekanntmachung Geeigneten Verhandlungen Der Konigliche Preussische Akademie Der Wissenschaften Zu Berlin, 57-97.

Ehrenberg, C. G. (1846). Über eine halibiolithische, von Herrn R. Schomburgk entdeckte, vorherrschend aus mikroskopischen Polycystinen gebildete, Gebirgsmasse von Barbados. Bericht Über Die Zur Bekanntmachung Geeigneten Verhandlungen Der Konigliche Preussische Akademie Der Wissenschaften Zu Berlin, 382-385.

Ehrenberg, C. G. (1847). Über die mikroskopischen Kieselschaligen Polycystinen als mächtige Gebirgsmasse von Barbados und über das Verhältnifs der aus mehr als 300 neuen Arten bestehenden ganz eigenthümlichen Formengruppe jener Felsmasse zu den jetzt lebenden Thieren und zur Kreidebildung. Eine neue Anregung zur Erforschung des Erdlebens. Bericht Über Die Zur Bekanntmachung Geeigneten Verhandlungen Der Konigliche Preussische Akademie Der Wissenschaften Zu Berlin, 40-60 + 1 pl.

Ehrenberg, C. G. (1854). Die systematische Characteristik der neuen mikroskopischen Organismen des tiefen Atlantischen Oceans für den Monatsbericht zum Druck $\mathrm{zu}$ übergeben, deren Verzeichnifs im Monat Februar bereits mitgetheilt worden ist. Bericht über die zur Bekanntmachung geeigneten Verhandlungen der Konigliche Preussische Akademie der Wissenschaften zu Berlin, 236-250.

Ehrenberg, C. G. (1860a). Über den Tiefgrund des stillen Oceans zwischen Californien und den Sandwich-Inseln aus bis 15600' Tiefe nach Lieutenant Brooke. Monatsberichte der Konigliche Preussische Akademie der Wissenschaften zu Berlin, 819-833.

Ehrenberg, C. G. (1860b). Über die organischen und unorganischen Mischungsverhaltnisse des Meeresgrundes in 19,800 Fuss Tiefe nach Lieut. Brookes Messung. Monatsberichte der Konigliche Preussische Akademie der Wissenschaften zu Berlin, 765-774.

Ehrenberg, C. G. (1861). Über die TiefgrundVerhaltnisse des Oceans am Eingange der Davisstrasse und bei Island. Monatsberichte der Konigliche Preussische Akademie der Wissenschaften zu Berlin, 275-315.

Ehrenberg, C. G. (1872a). Mikrogeologische Studien als Zusammenfassung seiner Beobachtungen des kleinsten Lebens der Meeres-Tiefgründe aller Zonen und dessen geologischen Einflufs. Monatsberichte Der Konigliche Preussische Akademie Der Wissenschaften Zu Berlin, 265-322.

Ehrenberg, C. G. (1872b). Mikrogeologischen Studien über das kleinste Leben der Meeres-Tiefgrunde aller Zonen und dessen geologischen Einfluss. Abhandlungen der Königlichen Akademie der Wissenschaften zu Berlin, 131-399 + tables and plates.

Ehrenberg, C. G. (1873). Grossere Felsproben des Polycystinen-Mergels von Barbados mit weiteren Erlauterungen. Monatsberichte Der Konigliche Preussische Akademie Der Wissenschaften Zu Berlin, 213-263.

Ehrenberg, C. G. (1875). Fortsetzung der mikrogeologischen Studien als 
Gesammt- Uebersichtder mikroskopischen Palaontologie gleichartig analysirter Gebirgsarten der Erde, mit specieller Rucksicht auf den PolycystinenMergel von Barbados. Abhandlungen der Königlichen Akademie der Wissenschaften zu Berlin, 1-225.

Foreman, H. P. (1973). 18. Radiolaria of Leg 10 with Systematics and Ranges for the Families Amphipyndacidae, Artostrobidae, and Theoperidae. Initial Reports of the Deep Sea Drilling Project, 10, 407-474. doi: 10.2973/dsdp.proc.10.118.1973

Foreman, H. P. (1975). 23. Radiolaria from the North Pacific, Deep Sea Drilling Project. Initial Reports of the Deep Sea Drilling Project, 32, 579-676. doi: 10.2973/dsdp. proc.32.123.1975

Forster, B., van de Ville, D., Berent, J., Sage, D. \& Unser, M. (2004). Complex wavelets for extended depth-of-field: a new method for the fusion of multichannel microscopy images. Microscopy Research and Techniques, 65(1-2), 33-42. doi: 10.1002/ jemt.20092

Goll, R. M. (1968). Classification and Phylogeny of Cenozoic Trissocyclidae (Radiolaria) in the Pacific and Caribbean Basins. Part I. Journal of Paleontology, 42(6), 1409-1432.

Goll, R. M. (1969). Classification and Phylogeny of Cenozoic Trissocyclidae (Radiolaria) in the Pacific and Caribbean Basins. Part II. Journal of Paleontology, 43(2), 322-339.

Goll, R. M. (1976). Morphological Intergradation between Modern Populations of Lophospyris and Phormospyris (Trissocyclidae, Radiolaria). Micropaleontology, 22(4), 379-418. doi: $10.2307 / 1485172$

Goll, R. M. (1979). The Neogene evolution of Zygocircus, Neosemantis, and Callimitra: their bearing on nassellarian classification. A revision of Plagiacanthoidae. Micropaleontology, 25(4), 365-396.

Goll, R. M. (1980). Pliocene-Pleistocene radiolarians from the East Pacific Rise and the Galapagos spreading center, Deep Sea Drilling Project Leg 54. Initial Reports of the Deep Sea Drilling Project, 54, 425453. doi: 10.2973/dsdp.proc.54.116.1980

Haeckel, E. H. P. A. (1860). Fernere Abbildungen und Diagnosen neuer Gattungen und Arten von lebenden Radiolarien des Mittelmeeres [Supplementary illustrations and diagnosis of new genera and species of living radiolarian of the Mediterranean Sea]. Monatsberichte der Konigliche Preussische Akademie der Wissenschaften zu Berlin, 835-845.

Haeckel, E. H. P. A. (1862). Die Radiolarien (Rhizopoda Radiaria). Berlin: Reimer.

Haeckel, E. H. P. A. (1881). Prodromus Systematis Radiolarium, Entwurf eines RadiolarienSystems auf Grund von Studien der Challenger-Radiolarien. Jenaische Zeitschrift Für Naturwissenschaft, 15(9, v.8), 418-472.

Haeckel, E. H. P. A. (1887). Report on the Radiolaria collected by H.M.S. Challenger during the years 1873-76. London: Eyre \& Spottiswoode, East Harding Street, Fetter Lane.

Harris, R. N., Sakaguchi, A., Petronotis, K., Baxter, A. T., Berg, R., Burkett, A., ... Zhao, X. (2013). Input Site U1414. Proceedings of the Integrated Ocean Drilling Program, 344, 1-87. doi: 10.2204/ iodp.proc.344.104.2013

Harting, P. (1863). Bijdrage tot de Kennis der mikroskopische Fauna en Flora van de Banda-Zee, naar aanleiding van een onderzoek van eenige door diepzeeloodingen van 990 tot 4000 
vademen uit die zee opgebragte gronden. Verhandelingen Der Koninklijke Akademie van Wetenschappen, 10(1), 1-34 + plates.

Haslett, S.K.(2002). Chapter 7.Palaeoceanographic applications of planktonik Sarcodine Protoza: Radiolaria and Foraminifera. In S. K. Haslett (Ed.), Quaternary Environmental Micropalaeontology (pp. 139-165). London: Arnold and Oxford University Press Inc.

Hollande, A. \& Enjumet, M. (1960). Cytologie, évolution et systématique des Sphaeroïdés (Radiolaires). Archives du Muséum National d'Histoire Naturelle, 7(VII), 1-134 + plates.

Johnson, D. A. \& Nigrini, C. A. (1980). Radiolarian biogeography in surface sediments of the western Indian Ocean. Marine Micropaleontology, 5(1), 111-152. doi: 10.1016/0377-8398(80)90008-0

Johnson, D. A. \& Nigrini, C. A. (1982). Radiolarian biogeography in surface sediments of the eastern Indian Ocean. Marine Micropaleontology, 7(3), 237-281. doi: $10.1016 / 0377-8398(82) 90004-4$

Jørgensen, E. G. (1900). Protophyten und Protozöen in Plankton aus der norwegischen Westküste. Bergens Museum Aarbog, $6,51-112+$ plates.

Jørgensen, E. G. (1905). The protist plankton of Northern Norwegian Fiords. In O. Nordgaard (Ed.), Hydrographical and Biological Investigations in Norwegian Fiords (pp. 49-151 + 13 plates). Bergens Museum Skrift: John Grieg.

Kamikuri, S., Motoyama, I., Nishi, H. \& Iwai, M. (2009). Neogene radiolarian biostratigraphy and faunal evolution rates in the eastern equatorial Pacific ODP Sites 845 and 1241. Acta Palaeontologica Polonica, 54(4), 713-742. doi: 10.4202/app.2008.0076
Kling, S. A. (1973). Radiolaria from the eastern North Pacific, Deep Sea Drilling Project, Leg 18. Initial Reports of the Deep Sea Drilling Project, 18, 617-671. doi: 10.2973/dsdp.proc.18.116.1973

Lazarus, D. (1990). 41. Middle Miocene to Recent radiolarians from the Weddell Sea, Antarctica, ODP Leg 113. Proceedings of the Ocean Drilling Program, Scientific Results, 113, 709-727. doi: 10.2973/odp. proc.sr.113.132.1990

Lazarus, D., Faust, K. \& Popova-Goll, I. (2005). New species of prunoid radiolarians from the Antarctic Neogene. Journal of Micropaleontology, 24, 97-121. doi: 10.1144/jm.24.2.97

Loeblich, J. A. R. \& Tappan, H. (1961). Remarks on the systematics of the Sarkodina [Protozoa], renamed homonyms and new validated genera. Proceedings of the Biological Society of Washington, 74, 213 234.

Martin, G. C. (1904). Radiolaria. In Miocene (pp. 447-459). Baltimore: The Johns Hopkins University Press.

Molina-Cruz, A. (1977). Radiolarian assemblages and their relationship to the oceanography of the subtropical southeastern Pacific. Marine Micropaleontology, $2(1), \quad 315-352$. doi: 10.1016/03778398(77)90016-0

Molina-Cruz, A. (1991). Holocene palaeo-oceanography of the northern Iceland Sea, indicated by Radiolaria and sponge spicules. Journal of Quaternary Science, 6(4), 303-312. https://doi.org/10.1002/ jqs. 3390060405

Moore Jr., T. C. (1971). Radiolaria. Initial Reports of the Deep Sea Drilling Project, 8, 727775. doi: 10.2973/dsdp.proc.8.112.1971 
Moore Jr., T. C. (1972). Mid-Tertiary evolution of the radiolarian genus Calocycletta. Micropaleontology, 18(2), 144-152. doi: $10.2307 / 1484991$

Moore Jr., T. C., van Andel, T. H., Sancetta, C. \& Pisias, N. G. (1978). Cenozoic hiatuses in pelagic sediments. Micropaleontology, 24(2), 113-138. doi: 10.2307/1485246

Müller, J. (1855). Über die im Hafen von Messinabeobachteten Polycystinen. Bericht über die zur Bekanntmachung geeigneten Verhandlungen der Konigliche Preussische Akademie der Wissenschaften zu Berlin, 671-676.

Müller, J. (1858). Über die Thalassicollen, Polycystinen und Acanthometren des Mittelmeeres. Abhandlungen der Königlichen Akademie der Wissenschaften zu Berlin, 1-62 + plates.

Nakaseko, K. (1963). Neogene Cyrtoidea (Radiolaria) from the Isozaki Formation in Ibaraki Prefecture, Japan. Science Reports, College of General Education, Osaka University, 12(2), 165-198.

Nigrini, C. A. (1967). Radiolaria in pelagic sediments from the Indian and Atlantic Oceans. Bulletin of the Scripps Institution of Oceanography of the University of California, 11, 1-125.

Nigrini, C. A. (1968). Radiolaria from Eastern Tropical Pacific Sediments. Micropaleontology, 14(1), 51-63. doi: $10.2307 / 1484765$

Nigrini, C. A. (1970). Radiolarian Assemblages in the North Pacific and Their Application to a Study of Quaternary Sediments in Core V20-130. In J. D. Hays (Ed.), Geological Investigations of the North Pacific, Geological Society of America Memoir (Vol. 126, pp. 139-183). doi: 10.1130/MEM126-p139
Nigrini, C. A. (1971). Radiolarian zones in the Quaternary of the equatorial Pacific Ocean. In B. M. Funnell \& W. R. Riedel (Eds.), The Micropoaleontology of Oceans: proceedings of the symposium held at Cambridge from 10-17 September 1967 (pp. 443-461). Cambridge (UK): Cambridge University Press.

Nigrini, C. A. (1977). Tropical Cenozoic Artostrobiidae (Radiolaria). Micropaleontology, 22(2), 241-269.

Nigrini, C. A. \& Lombari, G. (1984). A guide to Miocene radiolaria. Cushman Foundation for Foraminiferal Research, Special Publication, 22.

Nigrini, C. A. \& Moore Jr., T. C. (1979). A guide to modern radiolaria. Cushman Foundation for Foraminiferal Research, Special Publication, 16.

Nigrini, C. A. \& Sanfilippo, A. (2001). Cenozoic radiolarian stratigraphy for low and middle latitudes with descriptions of biomarkers and stratigraphically useful species. Ocean Discovery Program, Technical Notes, 27. Retrieved from http://www-odp.tamu.edu/ publications/tnotes/tn27/index.html

Petrushevskaya, M. G. (1965). Peculiarities of the construction of the skeleton of Radiolarians Botryoidae (Order Nassellaria). Transactions of the Institute of Zoology, 35, 79-118.

Petrushevskaya, M. G. (1971). On the natural system of polycystine Radiolaria (Class Sarcodina). In A. Farinacci (Ed.), Proceedings of the II Planktonic Conference (Vol. 2, pp. 981-992). Roma, Italy: Edizioni Tecnoscienza.

Petrushevskaya, M. G. (1975). 14. Cenozoic Radiolarians of the Antarctic, Leg 29, DSDP. Initial Reports of the Deep Sea 
Drilling Project, 29, 541-675. doi: 10.2973/dsdp.proc.29.114.1975

Petrushevskaya, M. G. (1981). Радиолярии отряда Nassellaria Мирового океанаа [Nassellarian radiolarians from the world ocean] (Izdavaemye, Zoologicheskim Institutom Akademii Nauk SSSR [Publications of the Zoological Institute, Academy of Sciences of the USSR], Vol. 128). Nauka, Leningradskoe Otdelenie, Leningrad, US.

Petrushevskaya, M. G., Cachon, J. \& Cachon, M. (1976). Comparative-morphological study of radiolarians: foundations of new taxonomy. Zoological Journal, 55, 485-496.

Petrushevskaya, M. G. \& Kozlova, G. E. (1972). Radiolaria: Leg 14, Deep Sea Drilling Project. Initial Reports of the Deep Sea Drilling Project, 14, 495-648. doi: 10.2973/ dsdp.proc.14.116.1972

Popofsky, A. (1908). Die Radiolarien des Antarktis mit Ausschluss Tripyleen. Tafel XXXXXVI, eine tabelle und 29 abbildungen im text. Deutsche Südpolar-Expedition 1901-1903, X, Zoologie II(1), 183-306.

Popofsky, A. (1913). Die Nassellarien des Warmwassergebietes der Deutschen Südpolar-Expedition 1901-1903. Deutsche Südpolar-Expedition 1901-1903, XIV, Zoologie VI(1), 217-416.

Popofsky, A. (1914). Die Collosphaeriden der Deutschen Südpolar-Expedition 19011903. Mit Nachtrag zu den Spumellarien und Nassellarien. Mit tafel XIII-XVII und 41 abbildungen im text. Part 1. Deutsche Südpolar-Expedition 1901-1903, XVI, Zoologie VIII(1), 235-278.

Principi, P. (1909). Contributo allo studio dei Radiolari Miocenici Italiani. Bollettino Della Societa Geologica Italiana, 28, 1-22.
Renz, G. W. (1976). The distribution and ecology of Radiolaria in the Central Pacific: plankton and surface sediments. La Jolla, California: University of California Press.

Riedel, W. R. (1957). Radiolaria: A preliminary stratigraphy. In H. Pettersson (Ed.), Sediment cores from the west Pacific (Vol. 6, pp. 59-96). Göteborg, Sweden: Elanders Boktryckeri Aktiebolag.

Riedel, W. R. (1959). Oligocene and Lower Miocene Radiolaria in tropical Pacific sediments. Micropaleontology, 5(3), 285-302.

Riedel, W. R. (1967). Some new families of Radiolaria. Proceedings of the Geological Society of London, 1640, 148-149.

Riedel, W. R. (1971a). Systematic classification of polycystine Radiolaria. In B. M. Funnell \& W. R. Riedel (Eds.), The Micropaleontology of Oceans: proceedings of the symposium held at Cambridge from 10-17 September 1967 (pp. 649-661). Cambridge (UK): Cambridge University Press.

Riedel, W. R. (1971b). The occurrence of pre-Quaternary Radiolaria in deep-sea sediments. In B. M. Funnell \& W. R. Riedel (Eds.), The Micropaleontology of Oceans: proceedings of the symposium held at Cambridge from 10-17 September 1967 (pp. 567-596). Cambridge (UK): Cambridge University Press.

Riedel, W. R. \& Sanfilippo, A. (1970). 24. Radiolaria, Leg 4, Deep Sea Drilling Project. Initial Reports of the Deep Sea Drilling Program, 4, 503-575. doi: 10.2973/dsdp.proc.4.124.1970

Riedel, W. R. \& Sanfilippo, A. (1971). Cenozoic Radiolaria from the western tropical Pacific, Leg 7. Initial Reports of the Deep Sea Drilling Project, 7, 1529-1672). doi: 10.2973/dsdp.proc.7.132.1971 
Riedel, W. R. \& Sanfilippo, A. (1978). Stratigraphy and evolution of tropical Cenozoic radiolarians. Micropaleontology, 24(1), 61-96. doi: $10.2307 / 1485420$

Riedel, W. R. \& Sanfilippo, A. (1986). Morphological characters for a natural classification of Cenozoic Radiolaria, reflecting phylogenies. Marine Micropaleontology, 11(1-3), 151-170. https://doi.org/10.1016/03778398(86)90011-3

Ryan, W. B. F., Carbotte, S. M., Coplan, J. O., O'Hara, S., Melkonian, A., Arko, R., ... Zemsky, R. (2009). Global MultiResolution Topography synthesis. Geochemistry Geophysics Geosystems, 10(3). doi: 10.1029/2008GC002332

Sachs, H. M. (1973). North Pacific Radiolarian assemblages and their relationship to oceanographic parameters. Quaternary Research, 3(1), 7388. doi: 10.1016/00335894(73)90054-9

Sandoval, M. I., Boltovskoy, D., Baxter, A. T. \& Baumgartner, P. O. (2017). Neogene paleoceanography of the eastern equatorial Pacific based on the radiolarian record of IODP drill sites off Costa Rica. Geochemistry Geophysics Geosystems, 18(3), 889-906. doi: 10.1002/2016GC006623

Sanfilippo, A. (1990). Origin of the subgenera Cyclampterium, Paralampterium and Sciadiopeplus from (Lophocyrtis) (Radiolaria, Theoperidae). Marine Micropaleontology, 15, 287-312.

Sanfilippo, A., Burckle, L. H., Martini, E. \& Riedel, W. R. (1973). Radiolarians, diatoms, silicoflagellates and calcareous nannofossils in the Mediterranean Neogene. Micropaleontology, 19(2), 209-234.
Sanfilippo, A. \& Riedel, W. R. (1970). PostEocene "Closed" Theoperid Radiolarians. Micropaleontology, 16(4), 442-462. doi: $10.2307 / 1485072$

Sanfilippo, A. \& Riedel, W. R. (1973). Cenozoic Radiolaria (exclusive of theoperids, artostrobiids and amphipyndacids) from the Gulf of Mexico, Deep Sea Drilling Project Leg 10. Initial Reports of the Deep Sea Drilling Project, 10, 475-611. doi: 10.2973/dsdp.proc.10.119.1973

Sanfilippo, A. \& Riedel, W. R. (1980). A revised generic and suprageneric classification of the Artiscins (Radiolaria). Journal of Paleontology, 54(5), 1008-1011. doi: 10.2307@130465

Schindlbeck, J. C., Kutterolf, S. O., Freundt, A., Alvarado, G. E., Wang, K.-L., Straub, S. M., ... Woodhead, J. D. (2016). Late Cenozoic tephrostratigraphy offshore the southern Central American Volcanic Arc: 1. Tephra ages and provenance. Geochemistry Geophysics Geosystems, 17(11), 46414668. doi: 10.1002/2016GC006503

Schindlbeck, J. C., Kutterolf, S. O., Freundt, A., Straub, S. M., Wang, K. L., Jegen, M., ... Sandoval Gutiérrez, M. I. (2015). The Miocene Galápagos ash layer record of Integrated Ocean Drilling Program Legs 334 and 344: Ocean-island explosive volcanism during plume-ridge interaction. Geology, 43(7), 599-602. doi: 10.1130/ G36645.1

Stöhr, E. (1880). Die Radiolarienfauna der Tripoli von Grotte, Provinz Girgenti in Sicilien. Palaeontographica, 22, series 3(2), 69124.

Suzuki, N. (2006). Ontogenic growth and variation in the skeletal structure of two late neogene Sphaeropyle species (Polycystina radiolarians). Journal 
of Paleontology, 80(5), 849-866. doi: 10.1666/0022-3360(2006)80[849:OGAVI $\mathrm{T}] 2.0 . \mathrm{CO} ; 2$

Takahashi, K. (1991). Radiolaria : flux, ecology, and taxonomy in the Pacific and Atlantic. Massachusetts, U.S.A.: Woods Hole Oceanographic Institution.

Takahashi, K. \& Honjo, S. (1981). Vertical Flux of Radiolaria: A Taxon-Quantitative Sediment Trap Study from the Western
Tropical Atlantic. Micropaleontology, 27(2), 140-190. doi: 10.2307/1485284

Tan, Z. \& Chen, M. (1999). Offshore Radiolaria in China. Beijing: Science Press.

van de Paverd, P. J. (1995). Recent Polycystine Radiolaria from the Snellius-II Expedition (Unpublished doctoral dissertation). Center for Marine Earth Science and Paleontological Museum in Oslo, The Netherlands \& Norway. 
The following figures illustrate the radiolarian with the sample code and the age assignment. In the supplementary data 1 and 2 are shown the entire samples list and the radiolarian abundance.
Some of the species could not be find in this counting because it was counted within a group. Each scale bar is $50 \mu \mathrm{m}$. 
Radiolaria Spumellaria

Figure 2

Family Collosphaeridae Müller, 1858

A. Acrosphaera sp. A. U1381C 7H 6W 23-25 (middle Miocene)

B. Acrosphaera sp. B U1381C 7H 3W 73-75 (middle Miocene)

C. Acrosphaera murrayana (Haeckel) U1414 A 1H 1W 2-4 (Pleistocene)

D-E. Siphonosphaera martensi Brandt, D. U1414 A 29X 3W 24-26, E. U1414 A 29X 3W 24-26 (late Miocene)

F-G. Siphonosphaera polysiphonia Haeckel, F. U1414 A 2H 3W 23-28 (Pleistocene)

G. U1414 A 10H 2W 24-26 (Pleistocene)

H-K. Collosphaera macropora Popofsky, H. U1414 A 28X 7W 24-26 (late Miocene), I. U1381C 6H 1W 39-41 (middle Miocene), J. U1381C 7H 3W 73-75 (middle Miocene), K. U1381C 7H 6W 60-62 (middle Miocene)

L. Collosphaera sp. A U1381C 7H 6W 60-62 (middle Miocene)

M. Collosphaera elliptica Chen and Tan U1381C 7H 6W 45-47 (middle Miocene)

N. Collosphaera tuberosa Haeckel U1414A 1H 1W 2-4 (Pleistocene)

O-Q. Otosphaera polymorpha Haeckel, O. U1414A 25X 5W 24-26 (late Miocene), P-Q. U1381C 7H 1W 119-121 (middle Miocene)

R-S. Otosphaera aff. auriculata, R. U1414A 6H 5W 26-27, S. U1414A 1H 1W 24-26 (Pleistocene)

T. Trisolenia zanguenica U1381C 7H 6W 45-47 (middle Miocene) 


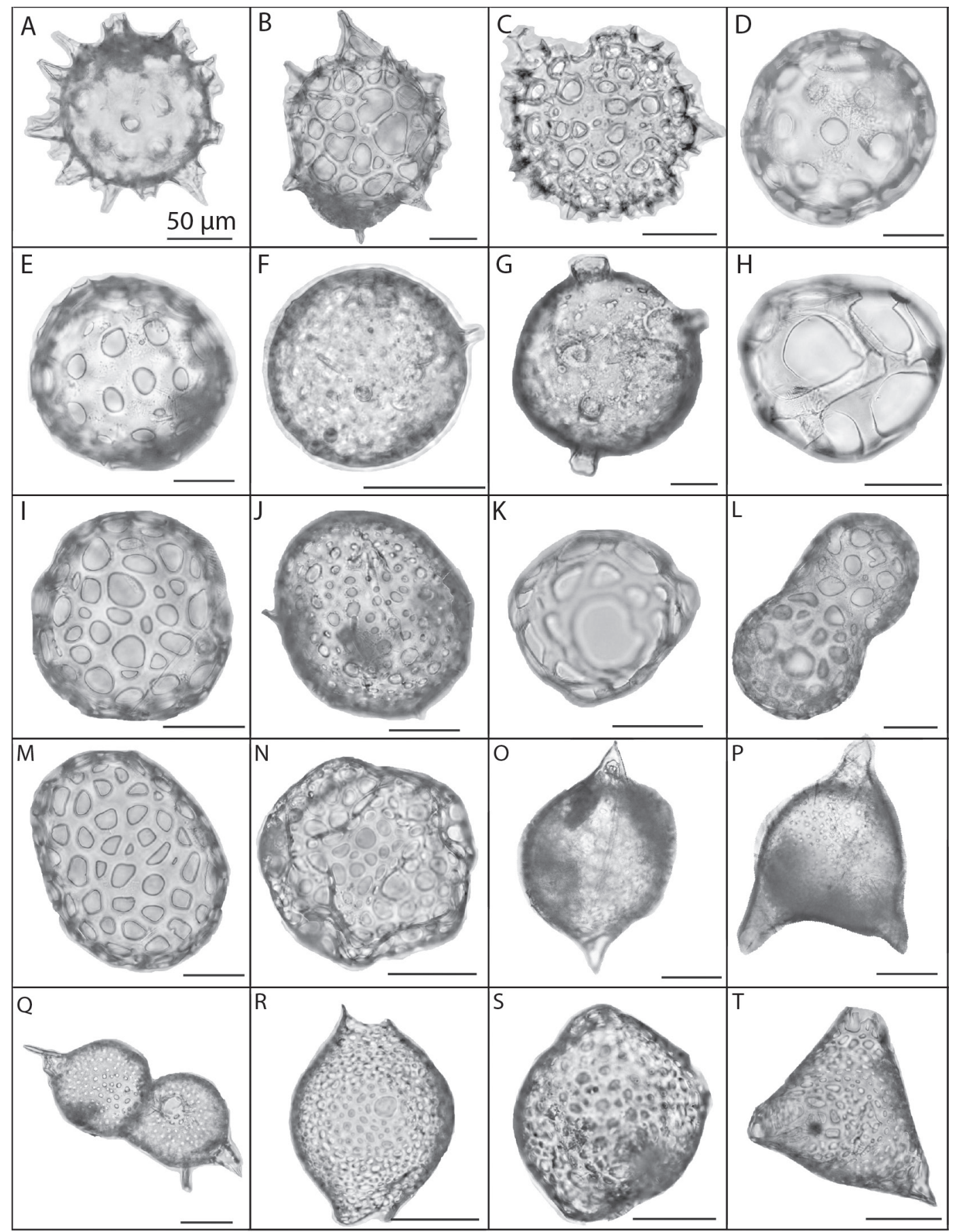


Figure 3

Family Actinommidae Haeckel, 1862, emend. Riedel, 1971

A-B. Actinomma langii (Dreyer), A. U1414A 2H 5W 17-22 (Pleistocene), B. U1414A 1H 1W 2-4 (Pleistocene)

C. Cladococcus cevicornis Haeckel U1414A 1H 1W 24-26 (Pleistocene)

D. Diartus hughesi (Campbell and Clark) U1414A 26X 7W 16-18 (late Miocene)

E-F. Diartus pettersoni Riedel and Sanfilippo, E. U1414A 31X 1W 24-26 (late Miocene), F. U1414A 29X 3W 24-26 (late Miocene)

G. Didymocyrtis penultima (Riedel) U1414A 22H 6W 24-26 (late Miocene)

H. Didymocyrtis antepenultima (Riedel and Sanfilippo) U1414A 27X 4W 24-26 (late Miocene)

I. Didymocyrtis laticonus (Riedel) U1381C 7H 2W 133-135 (late Miocene)

J. Didymocyrtis mammifera (Haeckel) U1381C 8H 3W 50-52 (late Miocene)

K. Didymocyrtis basanii (Carnevale) U1381C 7H 6W 45-47 (late Miocene)

L. Didymocyrtis tetrathalamus (Haeckel) U1414A 1H 1W 24-26 (Pleistocene)

M. Saturnalis circularis Haeckel U1381C 7H 6W 106-108 (middle Miocene)

N. Duppatractus sp. U1414A 30X 8W 24-36 (late Miocene)

O-P. Hexacontium armatum/hostile, O. U1414A 30X 5W 22-24 (late Miocene), P. U1381C 7H 3W

73-75 (middle Miocene)

Q. Stylosphaera sp. U1381C 7H 6W 106-108 (middle Miocene)

R. Plegmosphaera sp. U1414A 1H 1W 2-4 (Pleistocene)

Family Phacodiscidae Haeckel, 1881

S. Heliodiscus sp. U1381C 7H 1W 66-68 (middle Miocene)

Family Coccosdiscidae Haeckel, 1862

T. Periphaena decora U1414A 30X 8W 24-26 (late Miocene) 


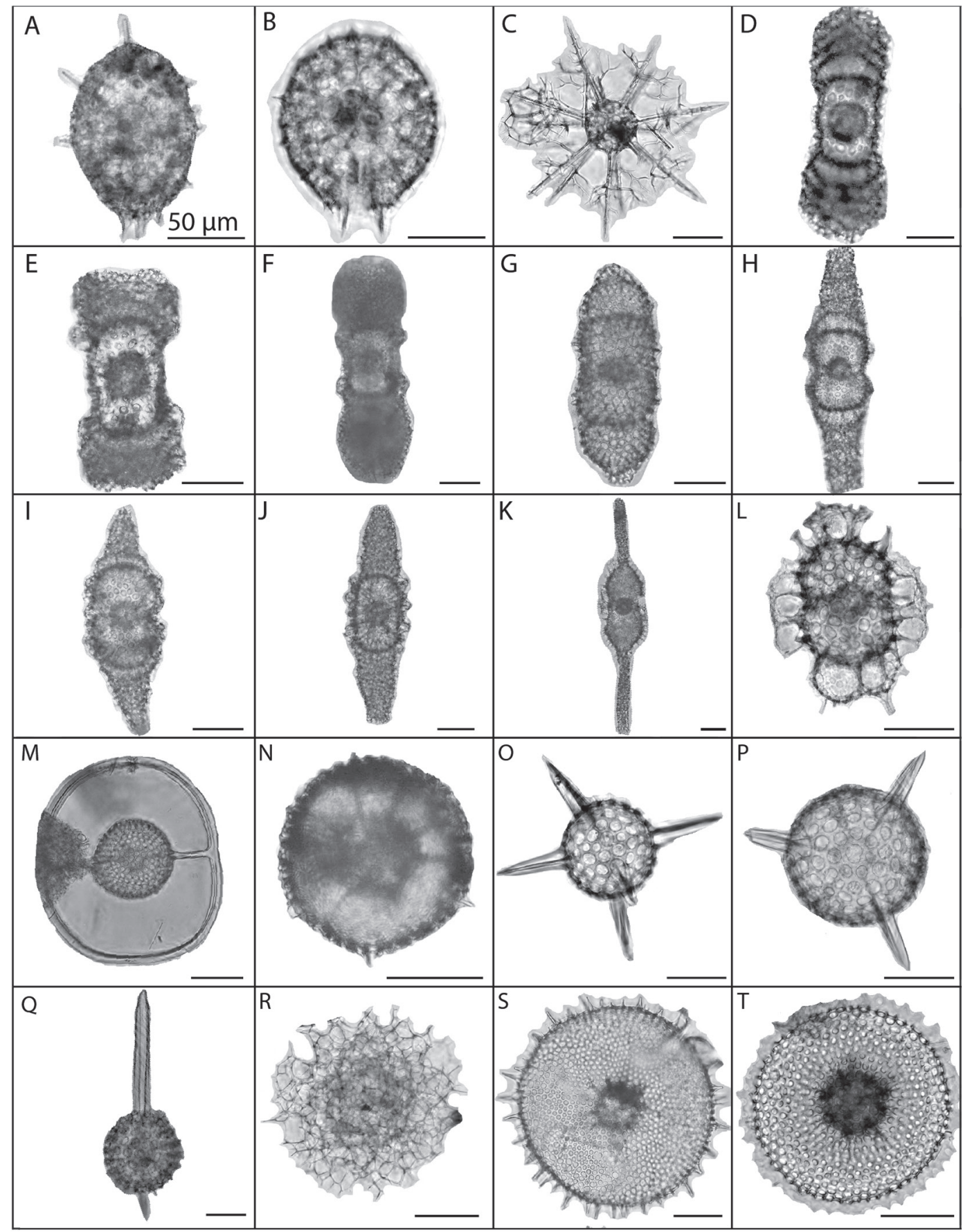


Figure 4

Family Spongodiscidae Haeckel, 1862, emend. Riedel, 1967a in Banner et al., 1967
A. Amphirhopalum ypsilum Haeckel U1414A 1H 1W 24-26 (Pleistocene)
B. Dictyocoryne truncatum (Ehrenberg) U1414A 1H 2W 2-4 (Pleistocene)
C. Dictyocoryne profunda Ehrenberg U1414A 1H 1W 24-26 (Pleistocene)

D-E. Euchitonia furcatalelegans group (Ehrenberg), D. U1414A 30X 5W 22-24 (Pleistocene), E. U1414A 4H 6W 24-26 (Pleistocene)

F. Spongaster tetras Ehrenberg U1414A 1H 1W 24-26 (Pleistocene)

G. Spongaster sp. A U1414A 7H 1W 137-139 (Pleistocene)

H. Spongocore puella Haeckel U1414A 3H 5W 24-26 (Pleistocene)

I. Stylodictya aculeata Jørgensen U1414A 26X 7W 16-18 (late Miocene)

J. Stylodictya validispina Jørgensen U1414A 30X 5W 22-24 (late Miocene)

K-L. Spongodiscus sp. K. U1414A 1H 1W 2-4, L. U1414A 1H 1W 2-4 (Pleistocene)

M-P. Circodiscus spp. M. U1414A 1H 1W 2-4 (Pleistocene), N. U1381C 7H 6W 45-47 (middle Miocene), O-P. U1414A 30X 5W 22-24 (late Miocene)

Q-S. Spongurus spp. Q-R. U1414A 1H 1W 2-4 (Pleistocene), S. U1414A 23X 6W 24-26 (late Miocene) T. Spongopyle osculosa U1414A 1H 1W 24-26 (Pleistocene) 


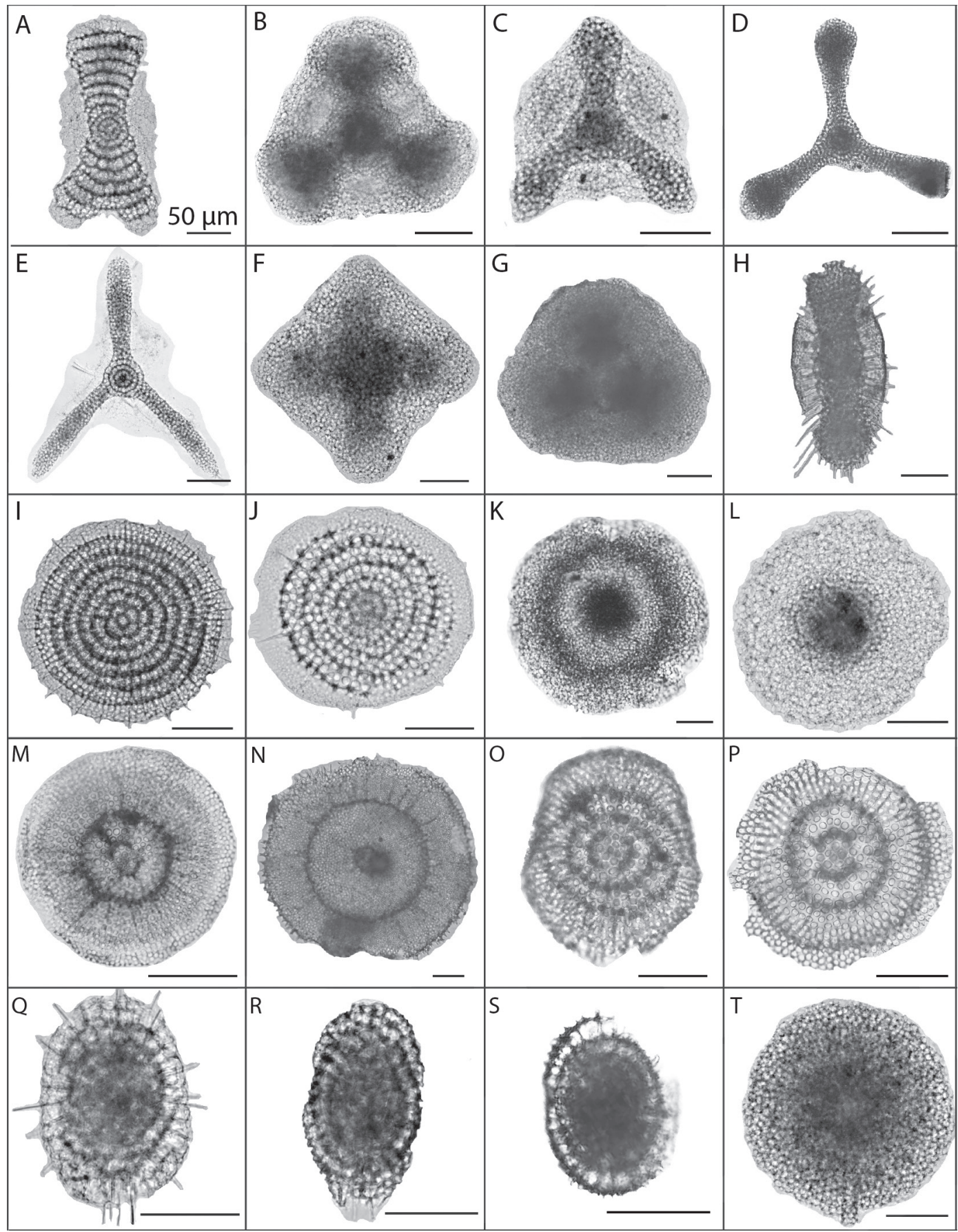


Figure 5

Family Spongodiscidae Haeckel, 1862, emend. Riedel, 1967a in Banner et al., 1967

A-C. Spongotrochus spp. A. U1414A 30X 4W, 20-22 cm, late Miocene) B-C. U1414A 1H 1W, 2-4 cm (Pleistocene)

Family Litheliidae Haeckel, 1882

D. Larcopyle butschlii Dreyer U1414A 1 H 1W 2-4 (Pleistocene)

E-F. Larcopyle nebulum Lazarus, E. U1381C 9H 4W 52-56 (middle Miocene), F. U1414 A 29X 3W 24-26 (late Miocene)

G-H. Lithelius minor (Jørgensen) U1414A 4H 3W 24-26 (Pleistocene)

I-J. Larcospira spp. U1414A 1H 1W 24-26 (Pleistocene)

K-L. Larcospira quandrangula Haeckel U1414A 1H 2W 22-24 (Pleistocene)

Family Pyloniidae Haeckel, 1881, emend. Dumitrica, 1989

M-O. Phorticium pylonium group Haeckel, M. U1414A 30X 5W 22-24 (late Miocene), N. U1414A 30X 6W 24-26 (late Miocene), O. U1414A 1H 1W 2-4 (Pleistocene)

P. Pylonium sp. sensu Benson U1414A 2H 5W 17-22 (Pleistocene)

Q. Tetrapyle octacantha Müller U1414A 6H 5W 26-27 (Pleistocene)

R-T. Spumell. gen and sp. indet. U1414A 1H 1W 24-26 (Pleistocene) 


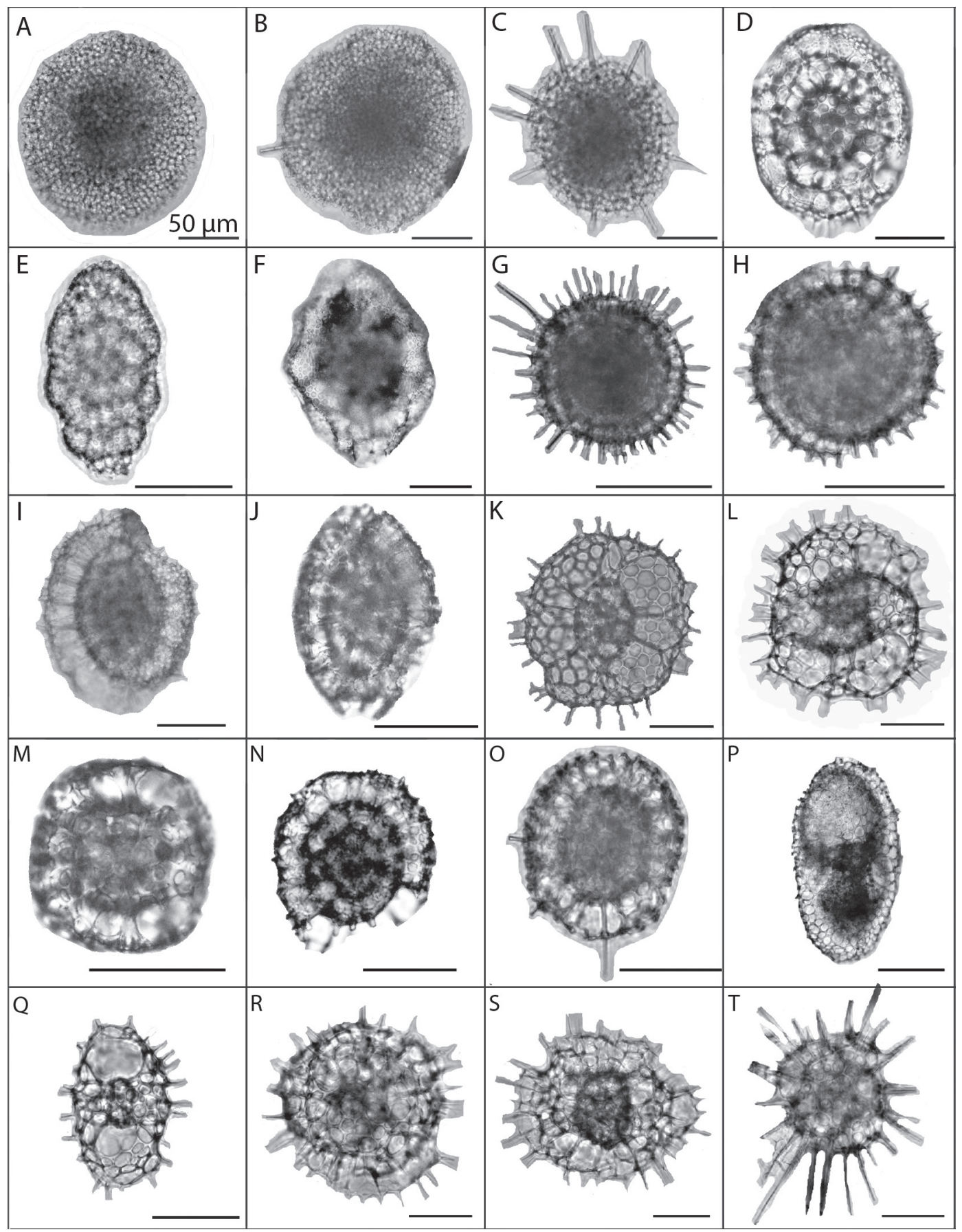


Radiolaria Nasellaria

Figure 6

Family Spyridae Ehrenberg, 1847, emend. Petrushevskaya, 1971

A. Amphispyris reticulata (Ehrenberg) U1414A 3H 1W 24-26 (Pleistocene)

B. Cantharospyris platybursa Haeckel U1414A 1H 2W 24-26 (Pleistocene)

C. Dendrospyris bursa Sanfilippo and Riedel U1414A 30X 7W 24-26 (late Miocene)

D. Giraffospyris toxaria (Haeckel) U1381C 7H 5W 126-128 (middle Miocene)

E. Liriospyris parkerae Riedel and Sanfilippo U1381C 7H 3W 28-30 (middle Miocene)

F-H. Liriospyris spp. F. U1414A 30X 5W 22-24 (late Miocene), G. U1381C 11H 1W 40-44 (middle Miocene), H. U1414A 31X 1W 24-26 (late Miocene)

I. Lophospyris pentagona pentagona (Ehrenberg) U1414A 1H 2W 24-26 (Pleistocene)

J. Neophrospyrs renilla Haeckel U1414A 6H 1W 26-27 (Pleistocene)

K. Androspyris huxleyi Haeckel U1414A 1H 1W 2-4 (Pleistocene)

L. Zigocircus productus Haeckel U1414 1H 1W 2-4 (Pleistocene)

M. Phormospyris sp. A U1414A 27X 4W 24-26 (late Miocene)

N. Phormospyris stabilis stabilis Goll U1414 1H 1W 24-26 (Pleistocene)

O. Phormospyris stabilis capoi Goll U1414A 5H 5W 24-26 (Pleistocene)

P. Phormospyris stabilis scaphipes (Haeckel) U1414A 4H 6W 24-26

Q-T. Tholospyris sp., Q. U1414A 30X 5W 24-26 (middle Miocene), R. U1381C 7H 3W 109-111 (middle Miocene), S-T. U1381C 7H 6W 106-108 (middle Miocene) 


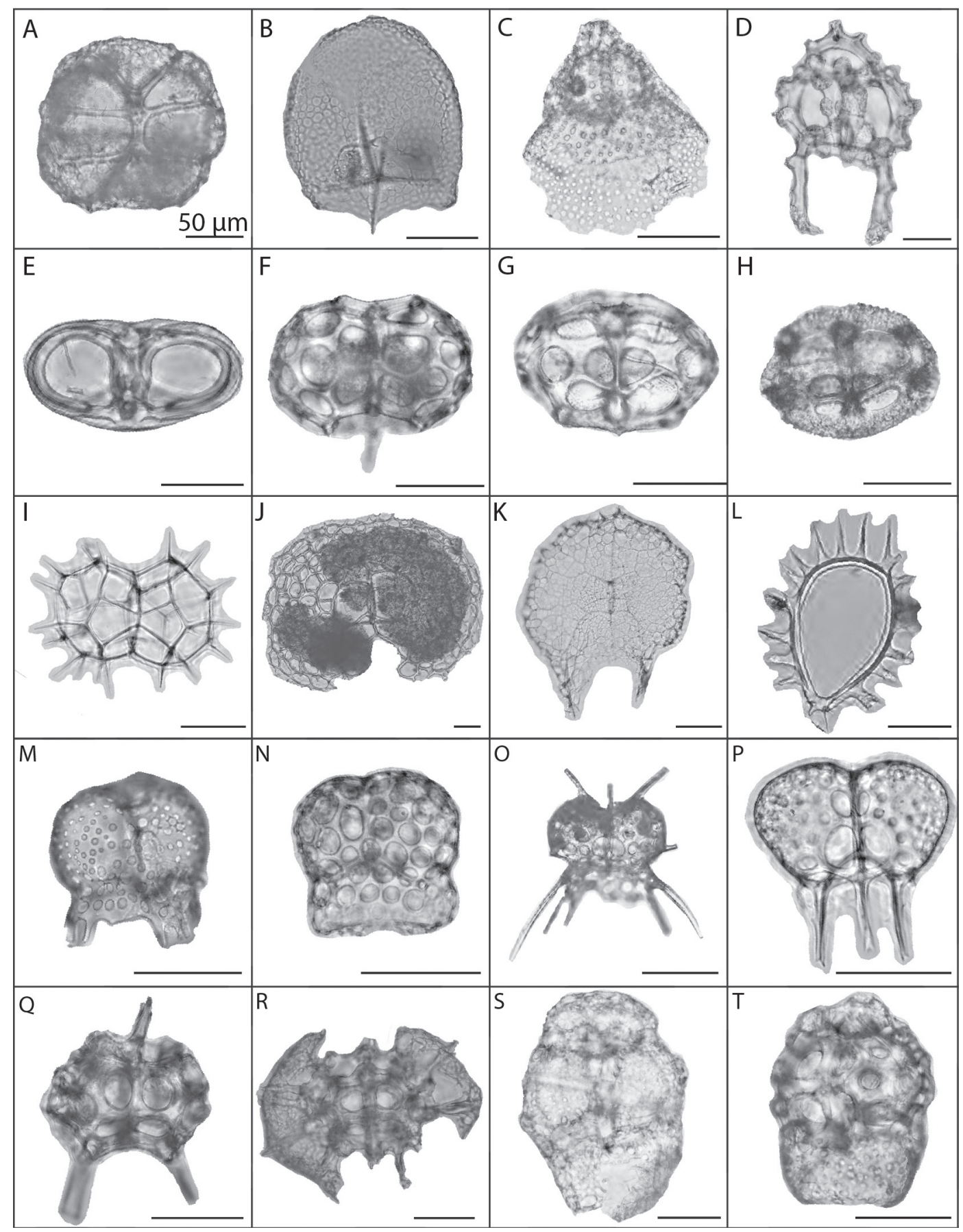


Figure 7

Family Plagoniidae Haeckel, 1881, emend. Riedel, 1967a in Banner et al., 1967
A. Amphiplecta acrostoma Haeckel U1414A 1H 1W 24-26 (Pleistocene)
B-D. Cladoscenium spp. B. U1414A 1H 1W 2-4 (Pleistocene), C. U1414A 1H 1W 24-26 (Pleistocene),
D. U1414 3H 1W 24-26 (Pleistocene)
E. Clathrocanium sp. U1381C 8H 3W 50-52 (middle Miocene)
F. Lophophaena hispida (Ehrenberg) U1414A 1H 2W 24-26 (Pleistocene)
G. Lophophaena aff. hispida U1414A 4H 6W 24-26 (Pleistocene)
H. Lophophaena aff. capito U1381C 7H1W 2-4 (middle Miocene)
I-L. Plagoniidae group, I. U1414A 1H 1W 2-4 (Pleistocene), J. U1414A 2H 3W 23-25 (Pleistocene),
K. U1381C 7H 6W 1-3, L. U1381C 7H 6W 23-25 (middle Miocene)
M. Pteroscenium sp. U1414A 1H 2W 24-26 (Pleistocene)
N. Pteroscenium pinnatum Haeckel U1414 29X 3W 24-26 (late Miocene)
O. Tetraphormis dodecaster (Haeckel) U1414A 1W 1H 2-4 (Pleistocene)
P. Clathrocorys aff. teuscheri U1414A 1H 1W 24-26 (Pleistocene)
Q-R. Helothus histricosa Jørgensen, Q. U1381C 7H 6W 23-25 (middle Miocene), R. U1414A 30X 5W 24-26 (late Miocene)
S. Helothus aff. histricosa U1381C 7H 6W 23-25 (middle Miocene)
T. Sethophormis sp.A U1381C 8H 3W 50-52 (middle Miocene) 


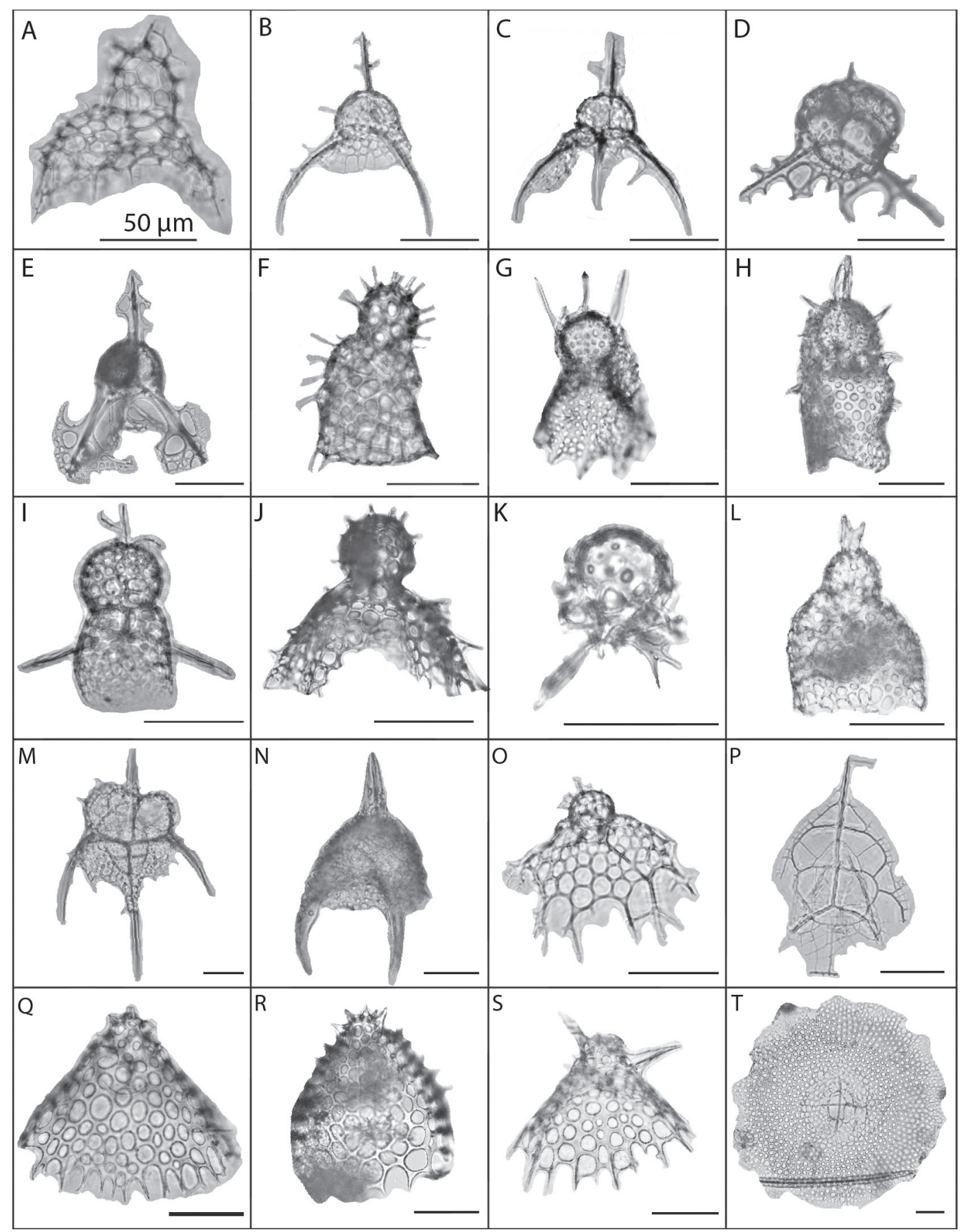


Figure 8

Family Theoperidae Haeckel, 1881, emend. Riedel, 1967a in Banner et al., 1967

A. Artopilium undulatum Popofsky U1414A 8H 1W 24-26 (Pleistocene)

B. Clathrocyclas alcmenae Haeckel U1414A 7H 2W 24-26 (Pleistocene)

C. Clathrocyclas monumentum Haeckel U1414A 1H 1W 24-26 (Pleistocene)

D-F. Corocalyptra kruegeri (Popofsky), D. U1381C 7H 6W 106-108 (middle Miocene), E. U1414A 30H 5W 22-24 (late Miocene), F. U1381C 7H 3W 4-6 (middle Miocene).

G. Corocalyptra sp. U1414A 2H 5W 17-22 (Pleistocene)

H. Corocalyptra cervus Ehrenberg U1414A 1H 1W 2-4 (Pleistocene)

I. Cornutella profunda Ehrenberg U1414 2W 5W 17-22 (Pleistocene)

J-K. Cycladophora davisiana (Ehrenberg), J. U1381C 2H 4W 66-68, K. U14141 A 1H 1W 24-26 (Pleistocene)

L-M. Cyrtocapsella cornuta Haeckel U1414A 27X 4W 24-26 (late Miocene)

N-O. Cyrtocapsella tetrapera (Haeckel) N. U1414A 30X 8W 24-26 (late Miocene), O. U1381C 11H IW 40-44 (middle Miocene)

P. Cyrtocapsella cylindroides (Principi) U1381C 7H 1W 137-139 (middle Miocene)

Q-S. Cyrtocapsella japonica (Nakaseko), Q-R. U1414A 30X 7W 24-26 (late Miocene), S. U1414A 30X 8W 24-26 (middle Miocene)

T. Cyrtocapsella sp. U1381C 7H 1W 137-139 (middle Miocene) 


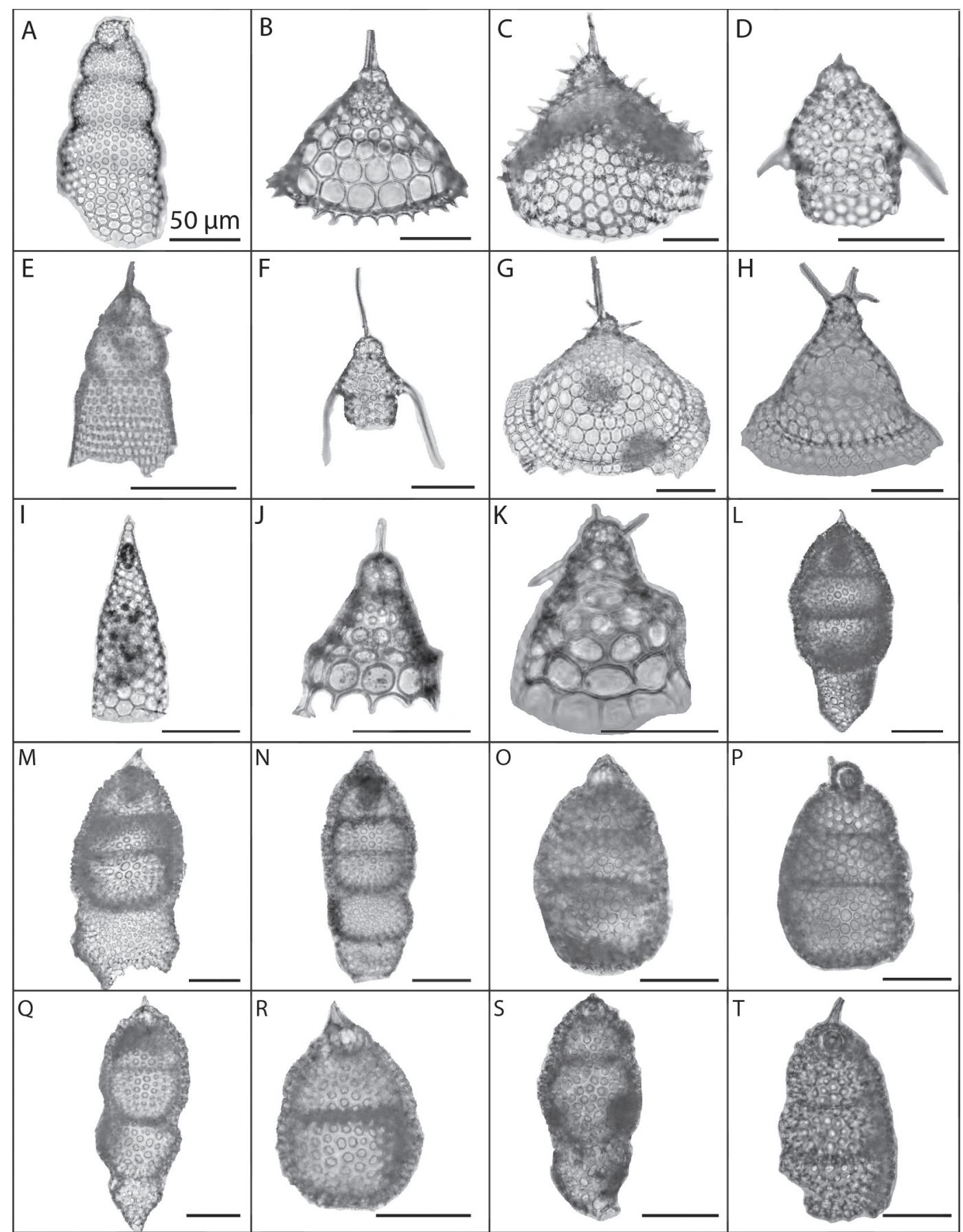


Figure 9

Family Theoperidae Haeckel, 1881, emend. Riedel, 1967a in Banner et al., 1967

A-B. Dictyophimus infabricatus Nigrini, A. U1414A 1H 1W 2-4 (Pleistocene), B. U1414A 1H 1W 24-26 (Pleistocene)

C. Dictyophimus hirundo Haeckel U1414A 1H 2W 24-26 (Pleistocene)

D-F. Dictyophimus spp. D. U1414A 1H 1W 24-26 (Pleistocene), E. U1414A 3H 1W 24-26 (Pleistocene), F. U1414A 30X 5W 22-24 (late Miocene)

G. Eucecryphalus gegenbauri Haeckel U1414A 1H 1W 2-4 (Pleistocene)

H-K. Eucecryphalus tricostatum Haeckel, H. U1414A 1H 2W 24-26 (Pleistocene), I. U1381C 7H 3W 28-30 (middle Miocene), J-K. U1414A 7H 2W 24-26 (late Miocene)

L-S. Eucecryphalus spp. L. U1381C 7H 1W 66-68 (middle Miocene), M. U1414A 30X 5W 22-24 (middle Miocene), N. U1381C 2H 4W 66-68 (Pleistocene), O. U1381C 7H 1W 37-39 (middle Miocene), P. U1414A 1H 1W 24-26 (Pleistocene), Q. U1381C 7H 3W 118-120, R-S. U1381C 5H 3W 89-91 (late Miocene), T. U1414A 29X 3W 24-26 (late Miocene) 


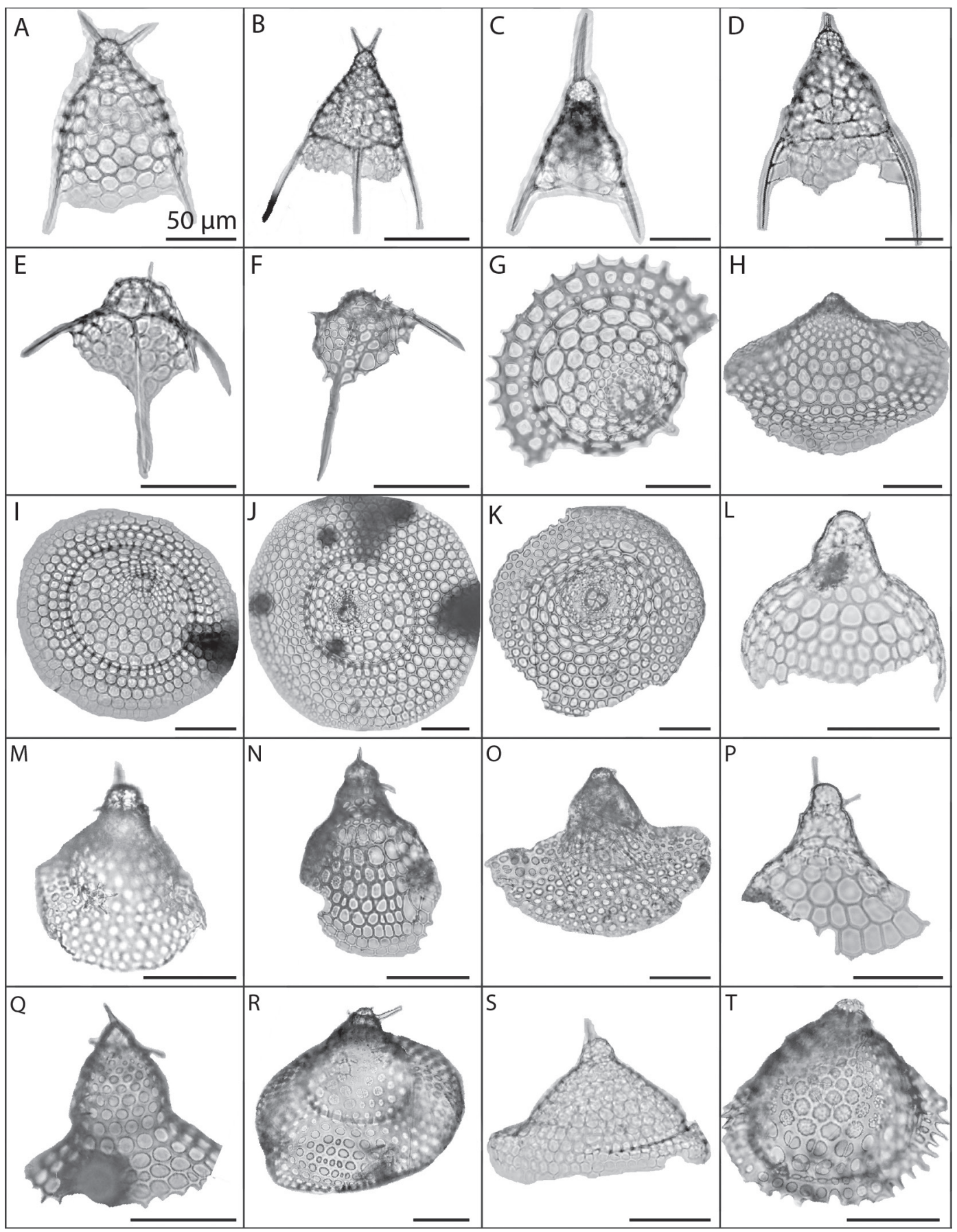


Figure 10

Family Theoperidae Haeckel, 1881, emend. Riedel, 1967a in Banner et al., 1967

A. Eucyrtidium anomalum (Haeckel) U1414A 24X 1W 24-26 (late Miocene)

B. Eucyrtidium calvertense Martin U1414A 22H 4W 24-26 (late Miocene)

C-D. Eucyrtidium cienkowskii Haeckel, C. U1414A 30X 5W 22-24 (late Miocene), D. U1381C 7H 1W 37-39 (middle Miocene)

E-G. Eucyrtidium hexagonatum Ehrenberg, E. U1414A 2H 5W 17-22 (Pleistocene), F. U1414A 1H 1W 2-4 (Pleistocene), G. U1414A 3H 1W 24-26 (Pleistocene)

H. Eucyrtidium hexastichus Haeckel U1381C 5H 1W 27-31 (late Miocene)

I. Eucyrtidium teuscheri Haeckel U1414A 3H 1W 24-26 (Pleistocene)

J-L. Eucyrtidium spp. J-K. U1414A 1H 1W 2-4, L. U1414A 2H 1W 17-22 (Pleistocene), M-N. U1414A 1H 1W 2-4 (Pleistocene), O. U1381C 5H 1W 27-31 (Pleistocene), P. U1414 28X 2W 2426 (late Miocene), Q-R. U1381C 7H 6W 106-108 (middle Miocene), S. U1381C 7H 6W 3W 73-75 (middle Miocene), T. U1381C 7H 6W 60-62 (middle Miocene). 


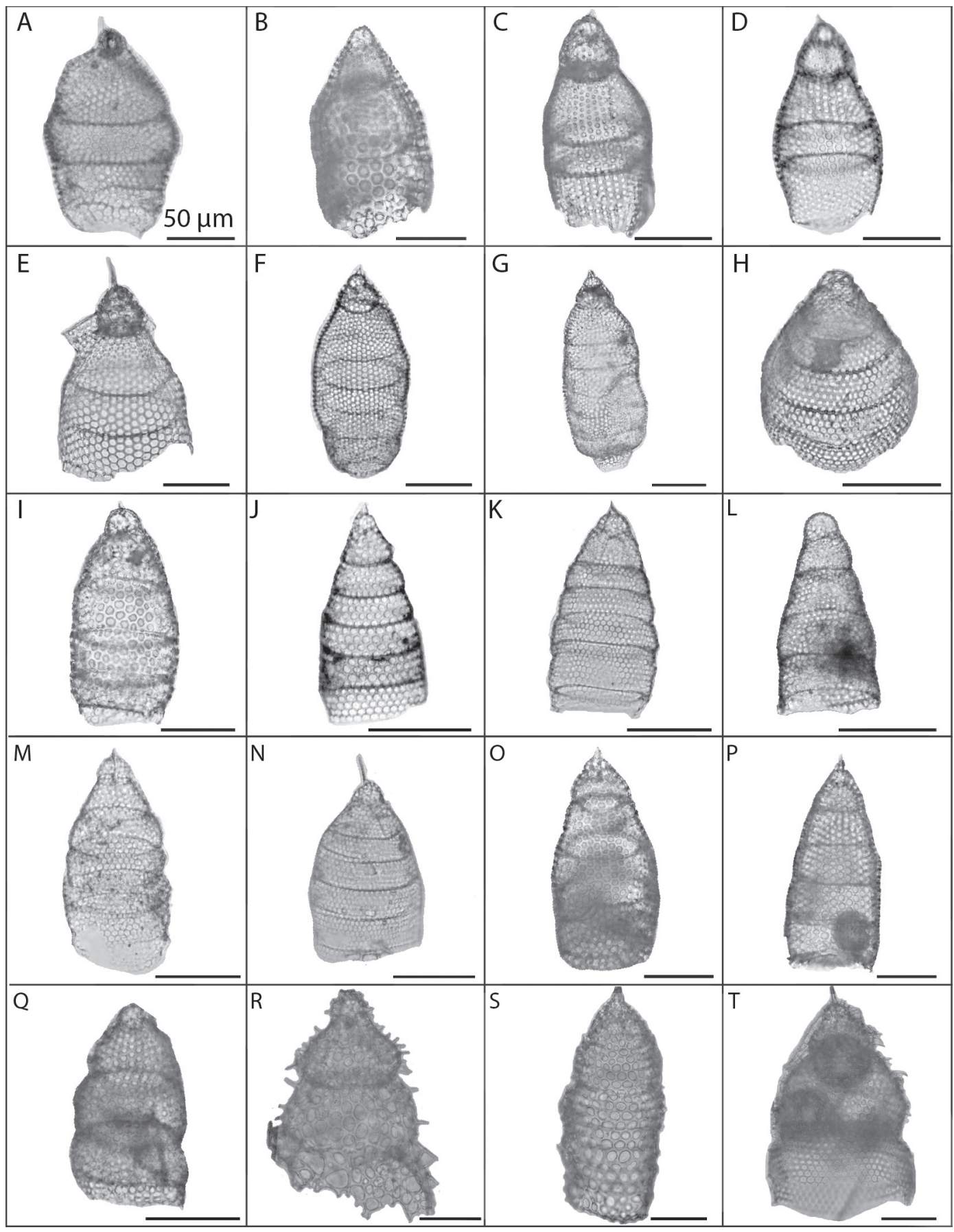


Figure 11

Family Theoperidae Haeckel, 1881, emend. Riedel, 1967a in Banner et al., 1967
A. Lampromitra schultzei Haeckel U1414A 1H 2W 24-26 (Pleistocene)
B. Litharachnium tentorium Haeckel U1414A 3H 5W 24-26 (Pleistocene)
C-D. Lipmanella dictyoceras (Haeckel), C. U1414A 4H 3W 24-26 (Pleistocene), D. U1381C 7H 2W 1-3 (middle Miocene)
E. Lipmanella sp. U1381C 10H 4W 36-40 (middle Miocene)
F-H. Lithopera neotera Sanfilippo and Riedel, F. U1414A 31X 1W 24-26 (late Miocene), G. U1414A 28X 7W 24-26 (late Miocene), H. U1414A 30X 7W 24-26 (late Miocene)

I-L. Lithopera renzae Riedel and Sanfilippo, I. U1381C 7H 3W 73-75 (middle Miocene), J. U1381C 7H 3W 73-75 (middle Miocene), K. U1414A 30X 8W 22-24 (late Miocene), L. U1381C 7H 6W 24-26 (middle Miocene)

M. Lithopera thornburgi Sanfilippo and Riedel U1414A 28X 7W 24-26 (late Miocene)

N. Lithostrobus hexagonalis Haeckel U1414A 4H 6W 24-26 (Pleistocene)

O. Lophocyrtis brachythorax (Sanfilippo and Riedel) U1414A 29X 3W 24-26 (late Miocene)

P. Lophocyrtis tanythorax Sanfilippo and Riedel U1381C 7H 1W 66-68 (middle Miocene)

Q-R. Lophocyrtis spp. Q. U1381C 7H 1W 119-121 (middle Miocene), R. U1381C 7H 1W 37-39 (middle Miocene)

S-T. Nassell. gen. and sp. indet., S. U1381C 9H 4W 52-56 (middle Miocene), T. U1381C 7H 3W 73-75 (middle Miocene) 


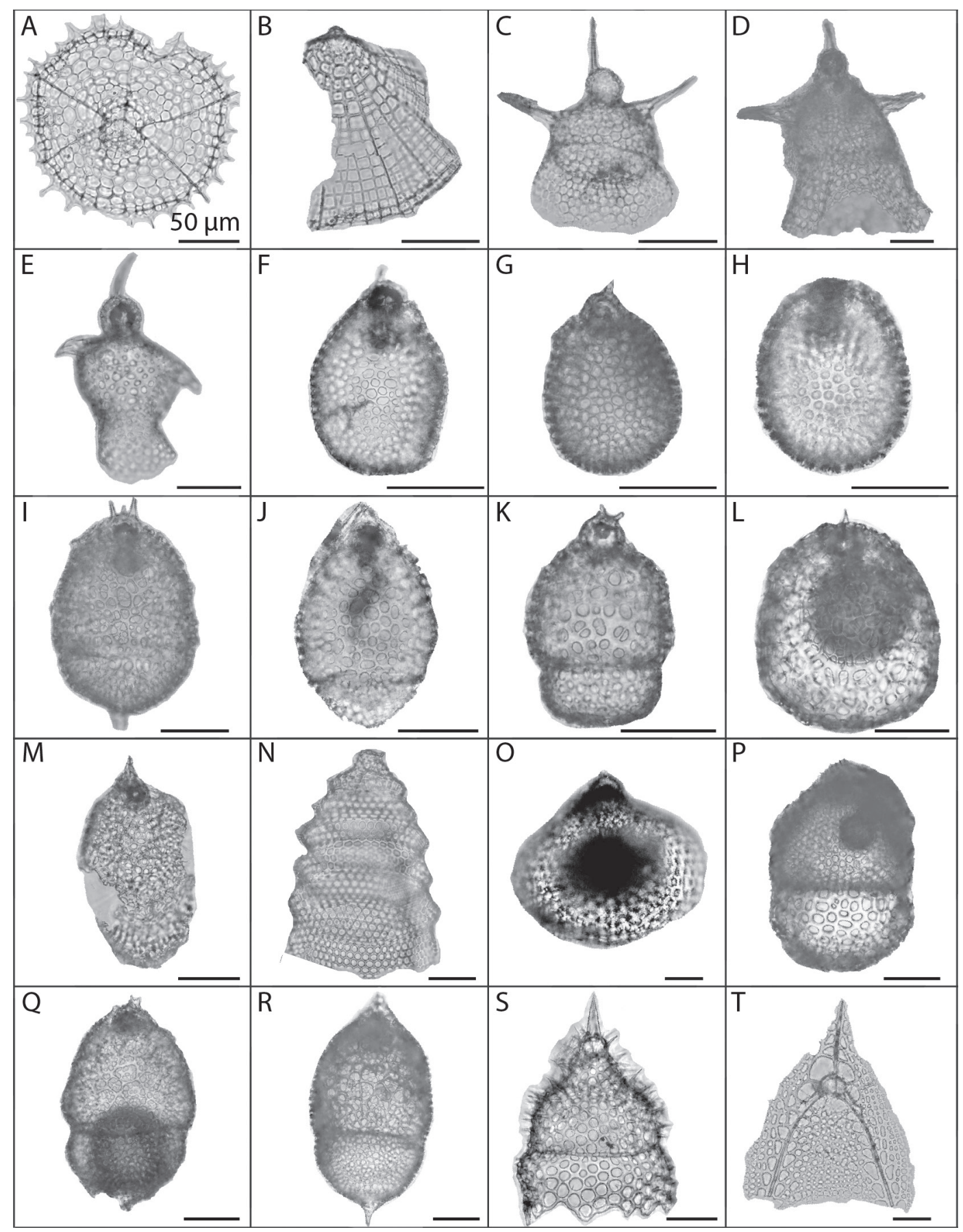


Figure 12

Family Theoperidae Haeckel, 1881, emend. Riedel, 1967a in Banner et al., 1967

A-B. Pterocanium grandiporus (Haeckel), A. U1414A 2H 5W 17-22 (Pleistocene), B. U1414A 3H 5W 24-26 (Pleistocene)

C-D. Pterocanium praetextum (Ehrenberg), C. U1414A 1H 1W 2-4 (Pleistocene), D. U1414A 1H 1W 24-26 (Pleistocene)

E-H. Pterocanium trilobum (Haeckel), E. U1414A 4H 6W 24-26 (Pleistocene), F. U1414A 10H 2W 24-26 (Pleistocene), G. U1414A 26X 7W 16-18 (late Miocene), H. U1414A 2H 5W 17-22 (Pleistocene) I-J. Stichocorys armata (Haeckel), I. U1381C 7H 1W 119-121 (middle Miocene), J. U1381C 7H 1W 119-121 (middle Miocene)

K-L. Stichocorys delmontensis (Campbell and Clark), K. U1381C 7H 1W 137-139 (middle Miocene), L. U1381C 7H 3W 28-30 (middle Miocene)

M. Stichocorys peregrina (Riedel) U1414A 31X 1W 24-26 (late Miocene)

N. Stichocorys sp. U1414A 30X 5W 22-24 (late Miocene)

O-P. Stichopilium bicorne Haeckel, O. U1414A 3H 1W 24-26 (Pleistocene), P. U1414A 3H 1W 24-26 (Pleistocene)

Q-S. Theocorys (?) spp. Q. U1381C 7H 6W 1-3 (middle Miocene), R. U1381C 7H 2W 1-3 (middle Miocene), S. U1381C 7H 1W 37-39 (middle Miocene).

T. Theocorys veneris (Haeckel) U1414A 1H 2W 24-26 (Pleistocene) 


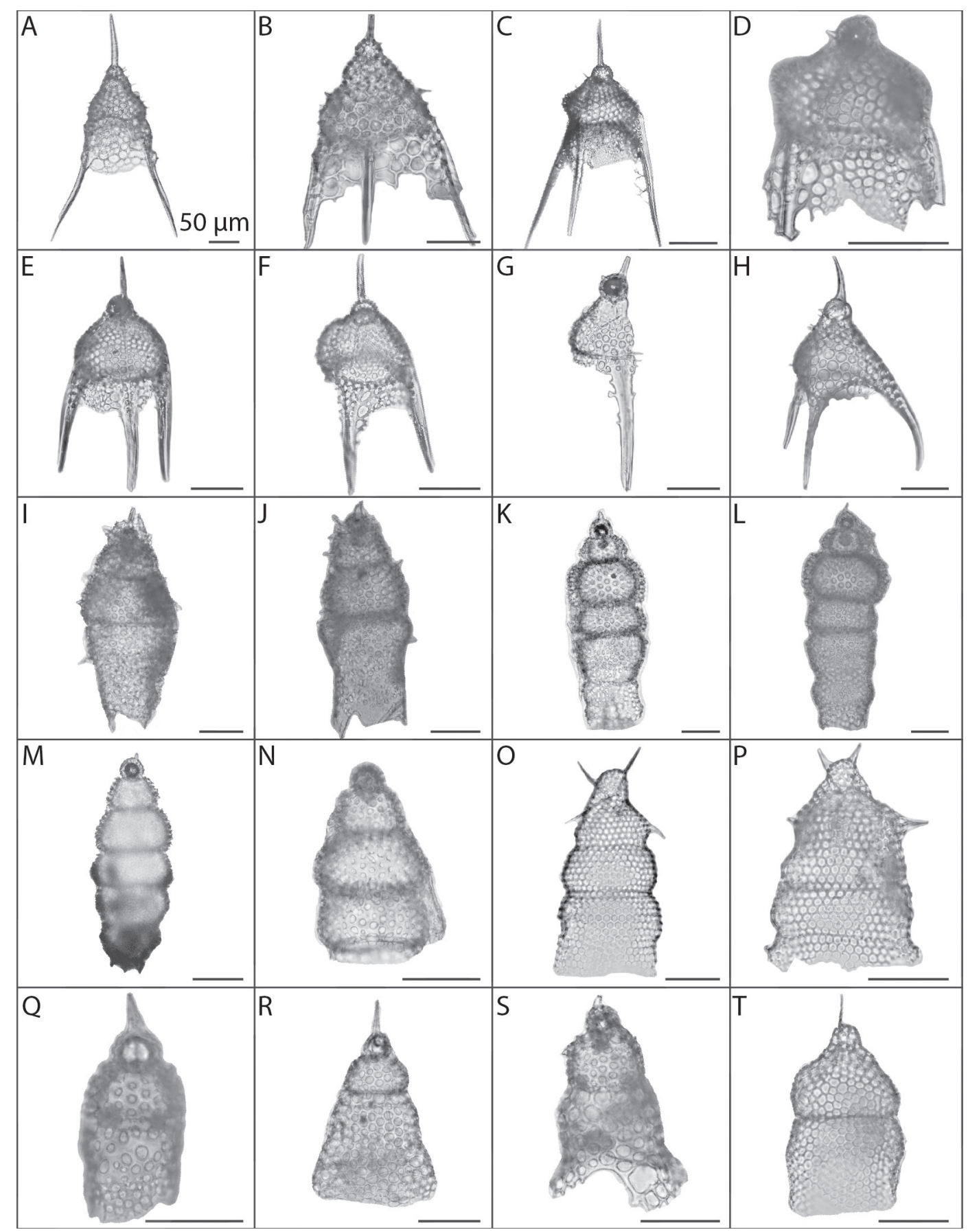


Figure 13

Family Theoperidae Haeckel, 1881, emend. Riedel, 1967a in Banner et al., 1967

A-B. Carpocanarium papillosum Ehrenberg, A. U1414A 27X 4W 24-26 (late Miocene), B. U1414A 28X 7W 24-26 (late Miocene)

C-D. Carpocanarium aff. papillosum, C. U1414A 29X 3W 24-26 (late Miocene), D. U1414A 30X 8W 24-26 (late Miocene)

Family Carpocaniidae Haeckel, 1881, emend. Riedel, 1967a in Banner et al., 1967

E-F. Carpocanistrum acutidentatum Takahashi, E. U1414A 2H 5W 17-22 (Pleistocene), F. U1414A 4H 6W 24-26 (Pleistocene)

G-I. Carpocanistrum spp. G. U1414A 3H 1W 24-26 (Pleistocene), H. U1414A 4H 6W 24-26 (Pleistocene), I. U1414A 10H 2W 24-26 (Pleistocene)

J-K. Carpocanopsis cristata (Carnevale), J. U1414A 31X 1W 24-26 (late Miocene), K. U1381C 7H 4W 52-56 (middle Miocene)

Family Cannobotryidae Haeckel, 1881

L-N. Botryocyrtis scutum (Harting), L. U1414A 2H 5W 17-22 (Pleistocene), 13M. U1414A 3H 5W 24-26 (Pleistocene), N. U1414A 30X 8W 24-26 (late Miocene)

O. Botryocyrtis sp. U1381C 5H 1W 27-31 (middle Miocene)

P-Q. Botryopyle dictyocephalus Haeckel U1381C 7H 2W 1-3 (middle Miocene)

R-S. Botryopyle sp. R. U1381C 7H 1W 119-121 (middle Miocene), S. U1381C 7H 3W 4-6 (middle Miocene)

T. Centrobotrys thermophila (Petrushevskaya) U1381C 7H 1W 37-39 (middle Miocene) 


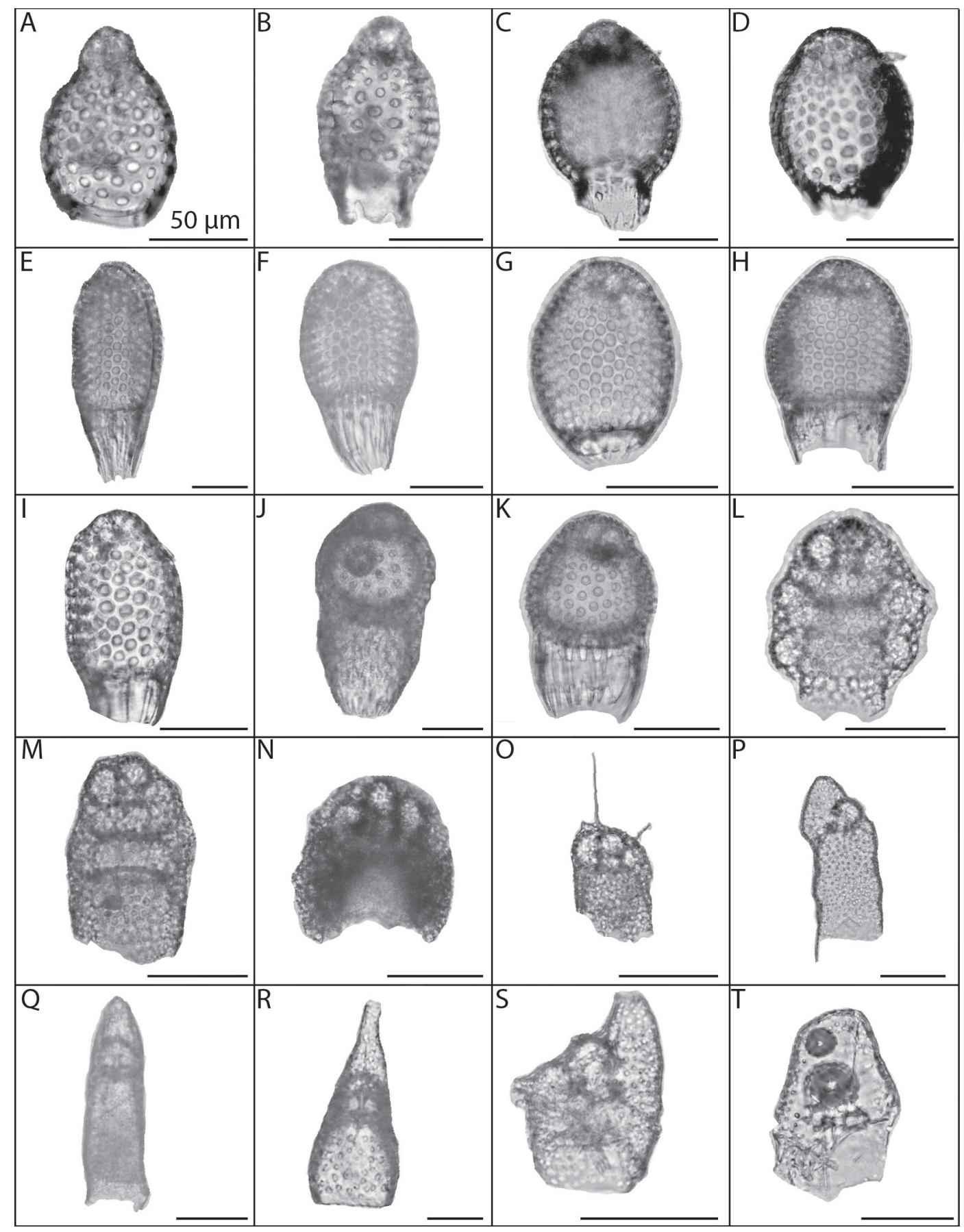


Figure 14

Family Artostrobiidae Riedel, 1967a in Banner et al., 1967 and Riedel, 1967b

A. Artostrobus annulatus (Bailey) U1414A 30X 5W 22-24 (late Miocene)

B-C. Botryostrobus aquilonaris (Bailey), B. U1414A 1H 1W 24-26 (Pleistocene), C. U1414A 3H 5W 24-26 (Pleistocene)

D-E. Botryostrobus auritus (Ehrenberg), D. U1414A 1H 2W 24-26 (Pleistocene), E. U1414A 4H 6W 24-26 (Pleistocene)

F. Botryostrobus bramlettei (Campbell and Clark) U1414A 30X 5W 22-24 (late Miocene)

G-H. Phormostichoartus corbula (Harting) U1414A 8H 1W 24-26 (Pleistocene)

I. Phormostichoartus doliolum (Riedel) U1414A 26 X 7W 16-18 (late Miocene)

J. Phosmostichoartus marylandicus (Martin) U1381C 7H 6W 60-62 (middle Miocene)

K-L. Siphocampe sp.A, K. U1414A 26X 7W 16-18 (late Miocene), L. U1381C 6H 7W 21-23 (middle Miocene).

M. Siphocampe arachnea (Ehrenberg) U1414A 30X 5W 22-24 (late Miocene)

N. Siphocampe lineata (Ehrenberg) U1381C 7H 6W 23-25 (middle Miocene)

O-P. Siphostichoartus corona (Haeckel), O. U1414A 26X 7W 16-18 (late Miocene), P. U1414A 30X 5W 22-24 (late Miocene)

Q. Spirocyrtis gyroscalaris Nigrini U1414A 28X 7W 24-26 (late Miocene)

R-S. Spirocyrtis scalaris Haeckel, R. U1414A 1H 2W 24-26 (Pleistocene), S. U1414A 1H 1W 2-4 (Pleistocene)

T. Spirocyrtis subtilis Petrushevskaya U1381C 7H 6W 23-25 (middle Miocene) 


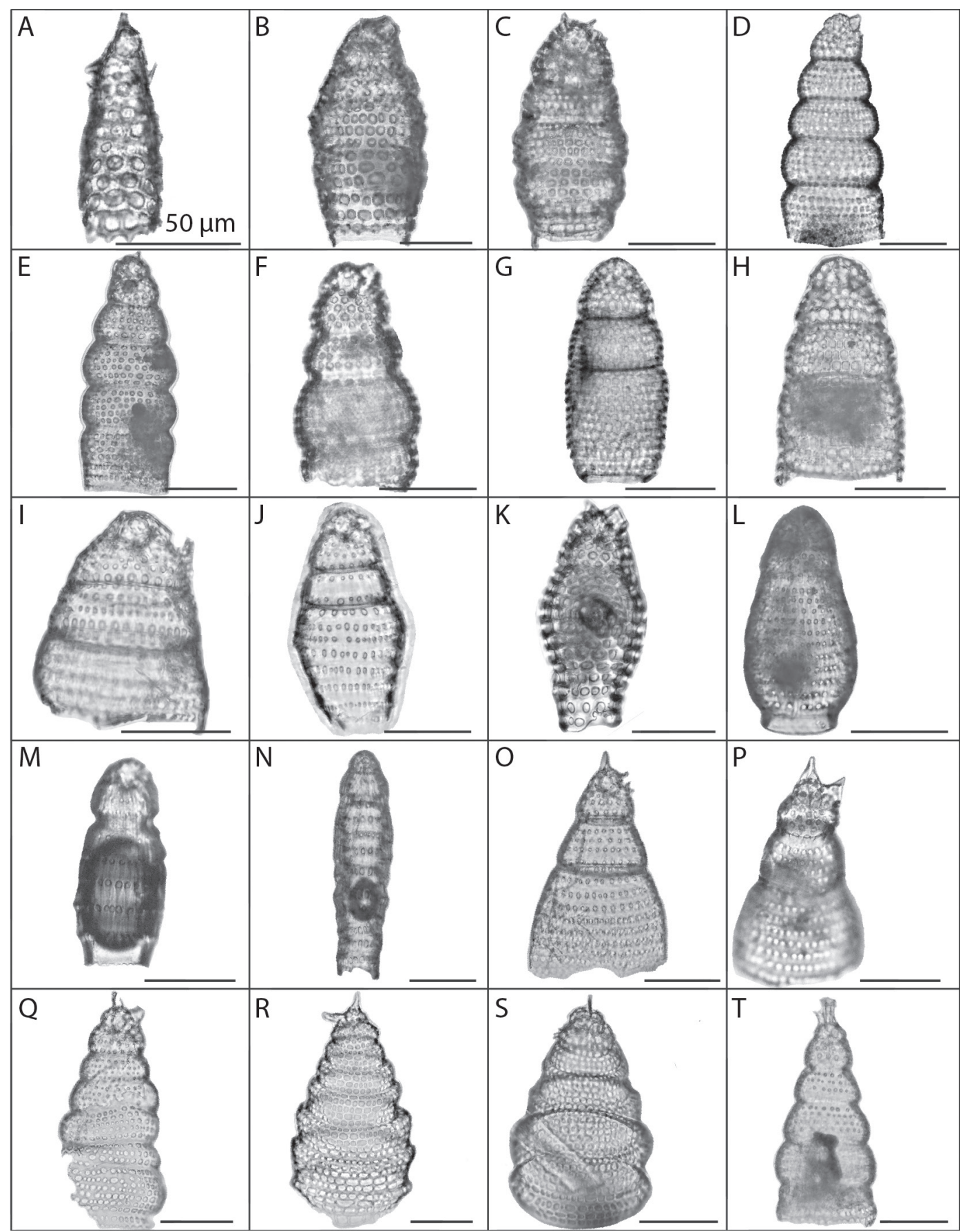


Figure 15

Family Pterocorythidae Haeckel, 1881, emend. Riedel, 1967a in Banner et al., 1967, emend. Moore, 1972

A-B. Anthocyrtidium ehrenbergi (Stöhr), A. U1381C 7H 5W 126-128 (middle Miocene), B. U1381C 7H 1W 37-39 (middle Miocene)

C-D. Anthocyrtidium ophirense (Ehrenberg), C. U1414A 3H 5W 24-26 (Pleistocene), D. U1414A 4H 6W 24-26 (Pleistocene)

E-F. Anthocyrtidium spp. E. U1414A 4H 6W 24-26 (Pleistocene), F. U1381C 7H 1W 137-139 (middle Miocene)

G-H. Calocycletta caepa Moore, G. U1381C 7H 6W 60-62, H. U1381C 7H 6W 106-108 (middle Miocene)

I. Calocycletta virginis Haeckel U1381C 7H 2W 133-135 (middle Miocene)

J. Lamprocyclas junonis (Haeckel) U1414A 7H 2W 24-26 (late Miocene)

K-L. Lamprocyclas maritalis Haeckel, K. U1414A 11H 3W 33-35 (Pleistocene), L. U1381C 7H 1W 137-139 (middle Miocene)

M. Lamprocyclas sp. A U1414A 28X 2W 24-26 (late Miocene)

N. Lamprocyrtis nigrinae Caulet U1414A 3H 5W 24-26 (Pleistocene)

O-P. Lamprocyrtis sp., O. U1414A 1H 1W 2-4, P. U1414A 4H 6W 24-26 (Pleistocene)

Q. Pterocorys minythorax (Nigrini) U1414A 1H 1W 24-26 (Pleistocene)

R-S. Pterocorys zancleus (Müller), R. U1414A 1H 1W 2-4 (Pleistocene), S. U1414A 1H 2W 24-26 (Pleistocene)

T. Pterocorys sp. U1414A 28X 2W 24-26 (late Miocene) 


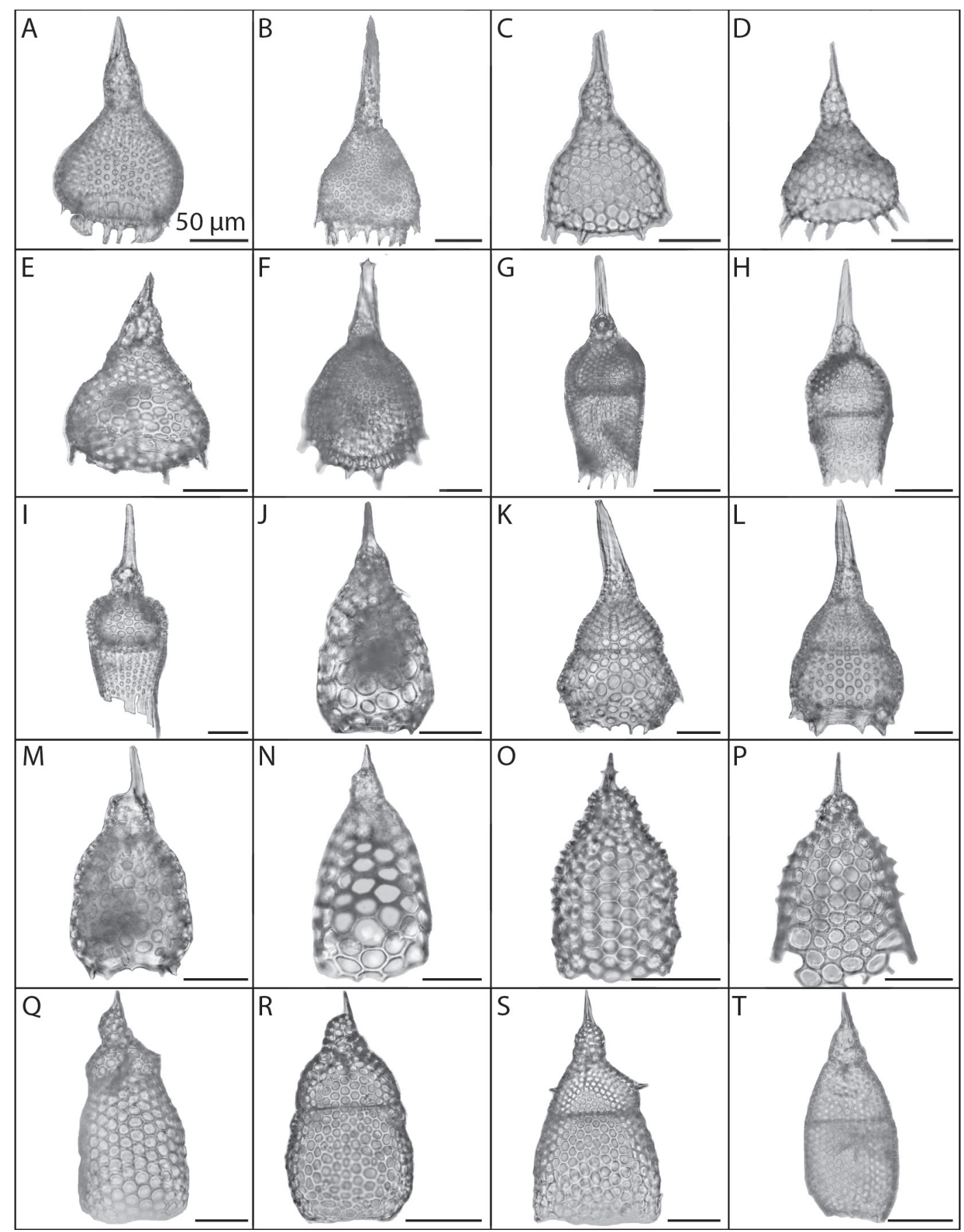

(i) (2) 2018 Universidad de Costa Rica. Revista Geológica de América Central is licensed under a Creative Commons AttributionNonCommercial-ShareAlike 3.0 Unported License. More information: http://www.geologia.ucr.ac.cr/revista/revista-geol.htm 
